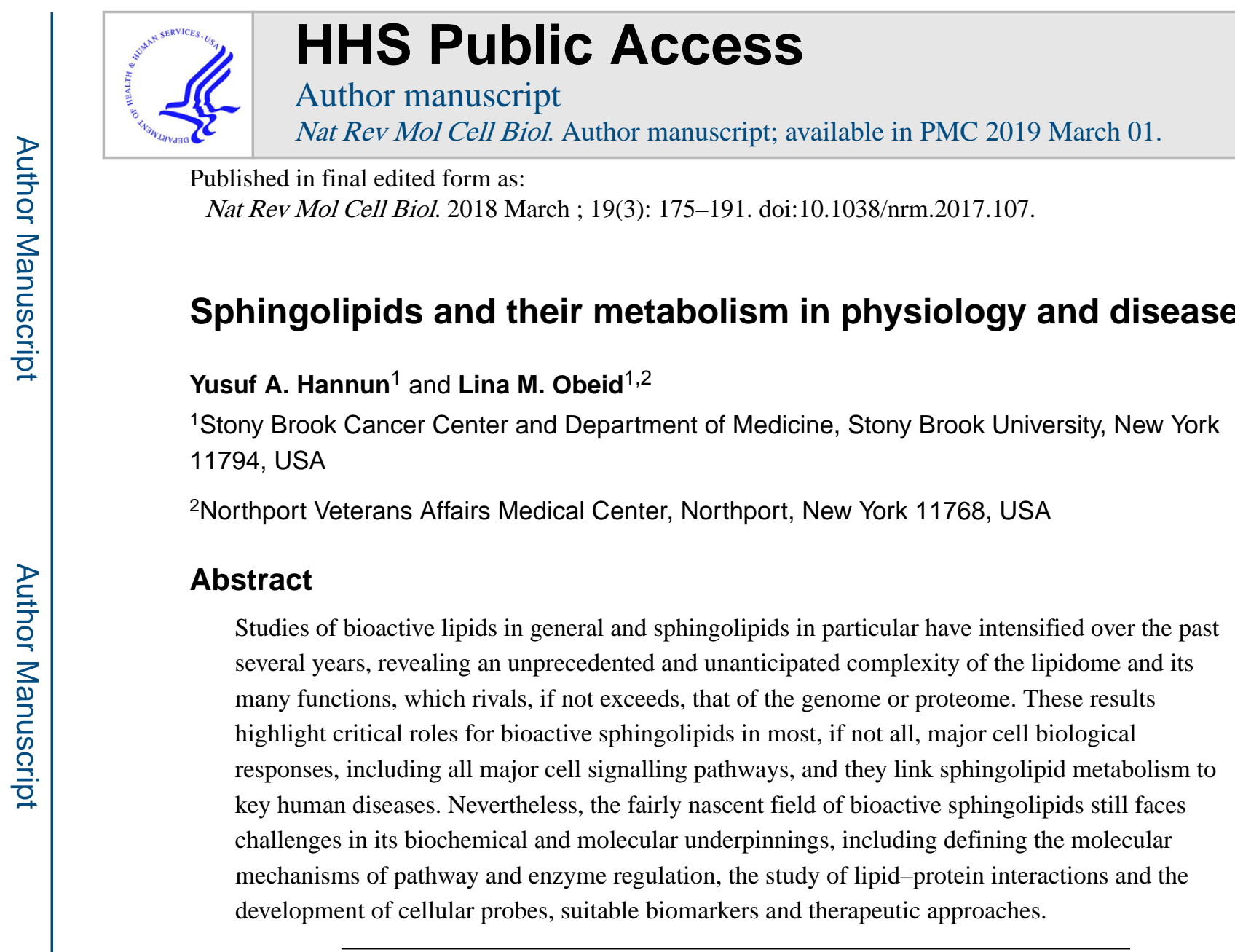

The concept of bioactive lipids and its utility for understanding cell and organismal biology has evolved over the past several decades, from studies on inositol phospholipids in the 1950s to studies on prostaglandins in the 1960s and the discovery of the bioactivity of diacylglycerol in the 1980s. This concept continues to gain considerable traction ${ }^{1}$. Bioactive lipids are functionally defined as lipid species, levels of which respond (acutely and/or tonically) to the action of specific stimuli. These lipids then regulate specific downstream effectors and targets. Thus, bioactive lipids are components of cellular regulatory circuits (such as signalling networks), which distinguishes them from other lipids that have structural and/or energetic functions.

We now understand that lipid metabolism is highly regulated and complex whereby many stimuli and agents affect one or more enzymes. The resulting changes in levels of lipids influence specific responses. Given the large number and diversity of these bioactive lipids and lipid-derived products (for example, diacylglycerol, phosphatidic acid, the various phosphatidylinositol phosphates, inositol trisphosphate, ceramides, sphingosine-1-phosphate

\footnotetext{
Author contributions

Both authors conducted extensive research of primary data and reviews for the article and collaborated in defining the scope and content of the Review, in writing the Review and in preparation of the figures and editing the manuscript.

Competing interests statement

The authors declare no competing interests.

SUPPLEMENTARY INFORMATION

See online article: S1 (box) |S2 (table) |S3 (table)

ALL LINKS ARE ACTIVE IN THE ONLINE PDF
} 
(S1P), sphingosine, cholesterol derivatives, eicosanoids and leukotrienes, to name a few), it is indeed nearly impossible for the cell biologist to understand any key cellular pathway without encountering one or more bioactive lipids. Yet, paradoxically, to the nonlipidologist, the universe of lipids, and especially sphingolipids, has been rather impenetrable owing to complexities in working with lipids at nearly all levels. Fortunately, important technological advances have enabled more researchers to study and probe pathways involving bioactive lipids (Supplementary information S1 (box)).

Sphingolipids represent one of the major classes of eukaryotic lipids. Historically, the first sphingolipids were isolated from brain in the late 19th century by Thudicum, who introduced the name 'sphingosin' after the Greek mythical creature, the Sphinx, in deference to "the many enigmas which it presented to the inquirer" (REF. 2). Biochemical and chemical approaches in the first part of the 20th century resulted in elucidation of the chemical structure of sphingosine, one of the major sphingoid bases, which are the founding blocks of all sphingolipids (distinguishing sphingolipids from other lipids). This discovery was followed by elucidation of the classes of complex sphingolipids (sphingomyelins and glycosphingolipids, including the gangliosides), which were appreciated as components of the plasma membrane and as modulators of cell-cell interactions and cell recognition. Concomitantly, sphingolipidoses — that is, defects in the metabolism of sphingolipids (details of which are shown in FIG. 1) - were discovered as lysosomal storage disorders in humans.

Studies in the mid-1980s defined bioactive functions for sphingosine, followed by ceramide and S1P. These emerging roles in various aspects of cell growth regulation catapulted sphingolipids into a verifiable class of bioactive lipids. The main bioactive sphingolipids (TABLE 1; Supplementary information S2 (table)) that have received the most attention are ceramide, sphingosine and S1P. Additional evidence also implicates ceramide-1-phosphate (C1P), glucosylceramide, lactosylceramide and some of the gangliosides as candidate bioactive lipids.

In this Review, we first present a brief background on bioactive sphingolipids and their metabolism, highlighting important progress made in the past decade. With several notable examples, we then illustrate the myriad physiological and pathophysiological roles of sphingolipids, providing evidence for their important functions in nearly all cell biological processes.

\section{Sphingolipid metabolism}

Bioactive sphingolipids are regulated by various enzymes and fluxes of different metabolites (FIG. 1), with around 40 enzymes in mammals involved in their metabolism (Supplementary information S3 (table)). These enzymes are the products of distinct genes, can occur as splice variants and can feature a plethora of post-translational modifications, which affect their enzymatic activity and function. Key regulated enzymes in de novo synthesis and hydrolysis are discussed in BOXES 1 and 2, respectively. The reader is also directed to recent reviews that extensively discuss metabolism of sphingolipids ${ }^{3-5}$. Critically, all key enzymes in sphingolipid metabolism have now been molecularly identified, which has 
revealed the vast complexity of these metabolic pathways and their precise subcellular compartmentalization.

\section{Box 1}

\section{Regulatory inputs and mechanisms of regulation of key enzymes involved in sphingolipid synthesis}

Serine palmitoyltransferase (SPT) serves as a critical node for regulating flux through sphingolipid metabolism ${ }^{158}$. Yeast $\mathrm{Spt}$ is a large protein complex comprising the main catalytic subunits Lcb1 (also known as Spt1) and Lcb2 (also known as Spt2) and regulatory proteins, including phosphoinositide phosphatase Sac1, the proteins Orm1 and Orm2 and the small subunit Spt-regulating protein Tsc3. Ceramide provides feedback inhibition through dephosphorylation of the Orms in a pathway involving protein phosphatase $2 \mathrm{~A}$ and its regulatory subunit Cdc55. Orms are further regulated by the action of the serine/threonine protein kinases Ypk and Pkh, downstream of target of

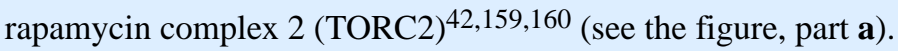

A wide range of stimuli have been shown to regulate the expression of individual ceramide synthases (CerSs) (refer to tavle 2 in REF. 11), and multiple mechanisms have been elucidated in the regulation of the CerSs. NAD-dependent protein deacetylase sirtuin 3, mitochondrial (SIRT3) directly deacetylates several CerSs, promoting CerS activity ${ }^{161}$. CerSs undergo casein kinase 2 (CK2)-dependent phosphorylation, which is suggested to increase CerS activity ${ }^{162}$. CerSs exist as homodimers and heterodimers, and their dimerization in the case of CerS2 and CerS6 is inhibited by binding to Bcl-2-like protein 13 (BCL2L13). This binding inhibits the activities of both synthases and consequently blocks apoptosis ${ }^{163}$. The pro-apoptotic protein Bcl-2 homologous antagonist/killer (BAK) activates CerS in vitro and in cells (by an unknown, indirect mechanism; see the figure, part $\mathbf{b}$, dashed arrow with question mark), indicating an intimate interaction between ceramide synthesis and apoptosis ${ }^{78}$. Various chemotherapeutic agents have been developed that promote CerS activity. In yeast, CerS is also regulated by phosphorylation downstream of TORC2 (see the figure, part b).

Sphingosine kinase 1 (SK1) is activated by growth factors, which, through downstream molecules such as protein kinase C (PKC) and phospholipase D (PLD), activate SK1 through the production of phosphatidic acid. SK1 can also be activated by phosphorylation, for example, through the kinases ERK1 and ERK2. SK1 mRNA is elevated in many cancers (partly owing to transcription activation through hypoxiainducible factor 1 (HIF1) and HIF2 downstream of hypoxic stress), and it also undergoes translational suppression by microRNAs (miRNAs) ${ }^{100}$, whereas SK1 protein levels are regulated by proteolysis. In the context of the DNA damage response (DDR), SK1 protein is digested by cathepsin in a p53-dependent mechanism ${ }^{68}$, whereas various pharmacological drugs (such as SKi, PF543 and ABC294640 (REF. 164)) can induce SK1 proteasomal degradation (see the figure, part c). SK1 activity is also influenced by its affinity for anionic membrane phospholipids and positive membrane curvature; these membrane-curvature-sensing properties of SK1 can be linked to its role in endocytosis ${ }^{64}$. 
These and additional (see also BOX 2) results disclose complex signalling networks and specific inputs that regulate fluxes through the sphingolipid pathway and/or levels of specific bioactive sphingolipids.

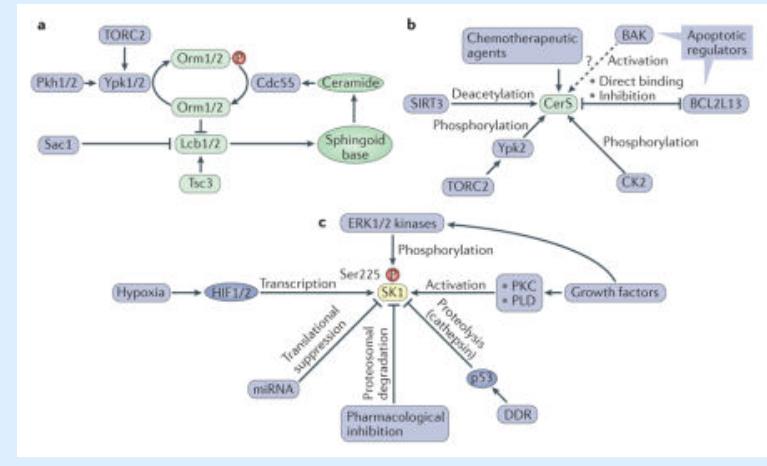

\section{Box 2}

\section{Mechanisms of regulation of key enzymes of sphingolipid catabolism}

Neutral sphingomyelinase 2 (nSMase2) is regulated at the mRNA level by p53 in the context of the DNA damage response (DDR) or by epigenetic mechanisms, including a combination of DNA methylation and histone deacetylation, with histone deacetylation mediating induction by all-trans retinoic acid (ATRA $)^{70,157}$. It is also regulated by serine phosphorylation promoted by reactive oxygen species (ROS) and tumour necrosis factor (TNF) signalling ${ }^{165}$. Mammalian nSMase has been shown to be allosterically activated by anionic phospholipids and to undergo palmitoylation on at least two distinct sites, which is critical for its association with the plasma membrane ${ }^{166}$ (see the figure, part a).

Acid sphingomyelinase (aSMase) is encoded by the gene SMPD1, and its mRNA exists as various splice variants, resulting in considerable structural variation in the protein ${ }^{167}$; regulatory factors of SMPD1 transcription are currently unknown. Translation generates a precursor protein that undergoes glycosylation in the Golgi and can then be targeted to the lysosome or for secretion ${ }^{127}$. The lysosomal form binds tightly to $\mathrm{Zn}$, which is required for enzymatic activity. Several cytokines, including TNF and interleukin-1 (IL-1), can induce the secretion of the enzyme (see the figure, part b) ${ }^{87}$. The activity of secreted aSMase is elusive, considering that the extracellular environment is not favourable for its action (neutral $\mathrm{pH}$, absence of $\mathrm{Zn}$ ).

Like nSMase, alkaline ceramidase 2 (ACER2) is also a target of p53-mediated activation in the context of the DDR, where it is responsible for the generation of sphingosine ${ }^{69}$. ACER2 is also induced by ATRA and by the synthetic retinoid fenretinide (4-HPR) ${ }^{168}$. By contrast, neutral ceramidase (NCDase) is transcriptionally inhibited by ATRA ${ }^{169}$ and by the cytotoxic drug gemcitabine, which induces NCDase proteolysis ${ }^{170}$. The mammalian sphingolipid 8(4)-desaturase DES1 (DEGS1) introduces the critical cis 4-5 double bond into the sphingoid backbone (which imparts apoptotic bioactivity to ceramide), and its inhibition attenuates ceramide and increases dihydroceramide levels, which has been implicated in the induction of autophagy ${ }^{76}$. DEGS1 activity is negatively 
regulated by ROS and inhibited by the direct action of 4-HPR ${ }^{171}$ and by tetrahydrocannabinol (THC) (see the figure, part c) ${ }^{76}$.

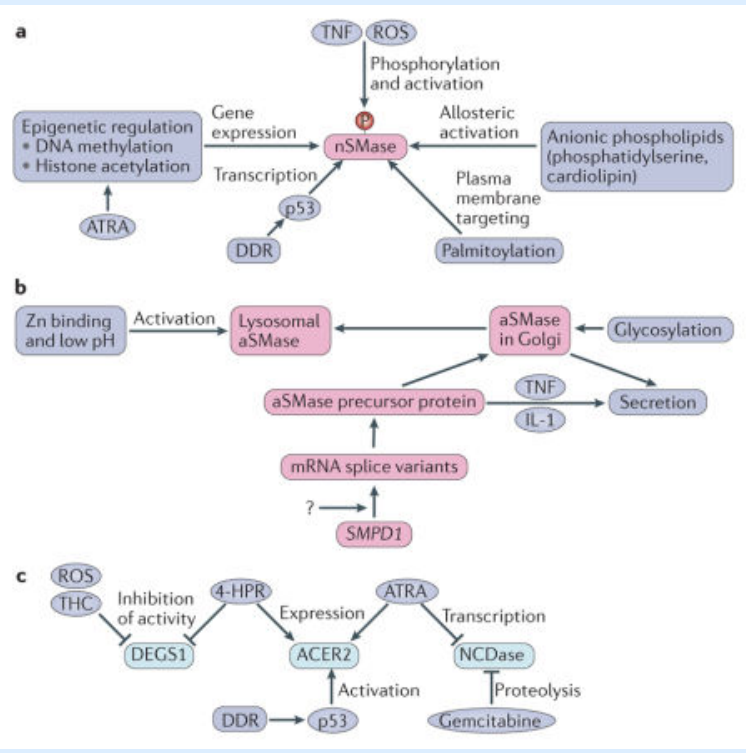

In its most basic conceptualization (FIG. 1A; TABLE 1; Supplementary information S2 (table)), the universe of sphingolipids can be thought of as a discrete set of metabolically connected pathways initiated by the condensation of the amino acid serine and the lipid palmitate. A set of enzymes then catalyses de novo synthesis, catabolism, recycling and interconversion of these lipids. Ultimately, the action of a lyase, acting on sphingoid base phosphates, provides the only known 'exit' from this network of sphingolipids (FIG. 1A).

This molecular era has allowed deep investigation of the functions of enzymes (an 'enzymecentric approach') through the use of contemporary tools of biochemistry, molecular biology and genetics, which has complemented a 'lipid-centric approach' that focuses on defining the function of individual bioactive lipids (Supplementary information S1 (box)). Focusing on enzymes has allowed deeper investigation into how these enzymes are regulated (BOXES 1,2 ), which in turn is essential for understanding their activities as well as the activities of their products - the various sphingolipid species (see below).

The use of model organisms together with sphingolipidomic and systems biology approaches also contributes to the dissection of the function and mechanisms of bioactive sphingolipids (Supplementary information S1 (box)). Importantly, mutations underlying various diseases have been found in several genes for enzymes of sphingolipid metabolism ${ }^{6}$ (Supplementary information S3 (table)). Together, these advances have enabled the establishment of prominent connections of bioactive sphingolipids to physiology and disease.

\section{Novel metabolic inputs, outputs and extensions}

Studies in the past decade have considerably advanced our understanding of several aspects of sphingolipid metabolism, including the entry of different substrates into sphingolipid 
metabolic pathways through serine palmitoyltransferase (SPT), the complexity of ceramide species and the exit from sphingolipid metabolism (FIG. 1A). They have also led to the discovery of previously unsuspected enzymes of sphingolipid metabolism.

Among the important novel findings is the demonstration that other substrates can enter sphingolipid metabolism, in addition to the canonically used serine and palmitate. This finding was initiated by the study of human hereditary sensory and autonomic neuropathy (HSAN1), which identified mutations in the genes encoding SPTLC1 and SPTLC2, the key subunits of SPT, as the main cause of this disease. Remarkably, these mutations alter the substrate specificity of SPT such that the mutants favour alanine and glycine over serine compared with wild-type forms of the enzyme $\mathrm{f}^{7}$. This results in the formation of noncanonical metabolites, the deoxysphingoid and 1-deoxymethyl sphingoid bases, respectively, and their acylated ceramides. Lacking the 1-hydroxyl (1-OH) moiety, both groups result in 'dead end' ceramides that cannot be modified at this position (FIG. 1B). These molecules have since been detected as normal products of SPT, albeit at very low levels. It is currently not known whether they have physiological functions, but they have been implicated as the mediators of neurotoxicity in HSAN1.

Additionally, the more recently identified SPTLC3 subunit of SPT confers specificity of the enzyme to myristate (over palmitate) as the fatty acid substrate ${ }^{8}$, whereas small subunits of SPT may favour the use of stearate as the fatty acid substrate ${ }^{9}$ (FIG. 1).

Thus, SPT is able to generate a multitude of sphingoid bases. Accordingly, inspired by molecular genetic nomenclature, SPT can be better dubbed Sag/PmsT to indicate its ability to use alanine and glycine as well as myristate and stearate, in addition to the canonical serine and palmitate, respectively.

These variations in the sphingoid backbone are compounded by variations in the acyl groups through the action of distinct ceramide synthases $(\mathrm{CerSs})^{10,11}$. Longer fatty acids are generated by the action of a family of elongases ${ }^{12}$ (FIG. 1A). Their combined action then allows a remarkable complexity in the structure of ceramides, which is further compounded by the action of additional hydroxylases and desaturases that act on either the sphingoid base or the fatty acyl group. Thus, ceramide is not a single species but should be considered a family of closely related but distinct molecules with emerging distinct functions (many ceramides instead of a single ceramide; see FIG. 1B for nomenclature) $)^{3}$.

More recent studies have also identified enzymatic activity involved in the $\omega$-acylation of ceramides to form three-chained ceramides and glycosphingolipids in the skin, which regulate epithelial permeability and skin barrier function (see below). The candidate genes include PNPLA1, which encodes patatin-like phospholipase domain-containing 1, which has phospholipase and transacylase functions ${ }^{13}$.

Ceramides can also be acylated at the 1-OH position, and a recent study identified their location in lipid droplets and the pathway leading to their formation involving a previously unrecognized ceramide acyl transferase activity of diacylglycerol O-acyltransferase 1 (DGAT1) and DGAT2 (REF. 14). It was also suggested that DGATs form a complex with CerS and with long-chain-fatty-acid-CoA ligase 5 (ACSL5), which acylates fatty acid 
substrates required for the action of DGATs. In related studies, the fatty acyl binding protein ACBP has been shown to specifically interact with CerS2 and CerS3 and to channel verylong-chain fatty acids to ceramide synthesis ${ }^{15}$. This indicates the important roles of protein complexes in controlling sphingolipid metabolism.

There have also been notable advances in following the fate of the fatty aldehydes as they exit the sphingolipid pathway. These studies defined the dehydrogenase ALDH3A2, CoA synthases ACSL1-6, ACSBG1 and ACSVL1 (also known as VLACS), and reductase TSC13 as the enzymes involved in sequential metabolism of the fatty aldehyde products of sphingolipid clearance ${ }^{16}$ (FIG. 1).

Two novel previously unsuspected enzymes were also identified that add to the complexity of sphingolipid metabolism. Sphingomyelin synthase-related (SMSr) was identified as a homologue of SMS but with much reduced activity and with a preference for utilizing phosphatidylethanolamine as a donor of ethanolamine phosphate and thus generating ceramide ethanolamine phosphate rather than sphingomyelin (where phosphatidylcholine serves as the donor of choline phosphate) ${ }^{17}$. Mitochondrial sphingomyelinase was identified as a predominantly mitochondrial neutral sphingomyelinase, the functions of which are currently unknown ${ }^{18}$.

These advances have provided a strong foundation for understanding the biochemistry of sphingolipid metabolism, illustrating its many complexities but also its integration in broader aspects of lipid metabolism and metabolomics in general. Understanding these enzymes and pathways has also opened up new avenues for the development of therapeutics targeting sphingolipid-related diseases (BOX 3; Supplementary information S3 (table)).

\section{Box 3}

\section{Therapeutic targeting of sphingolipids}

Pathways of sphingolipid metabolism have become important targets for the development of therapeutics. First, analogues of bioactive sphingolipids are proving to be of use in the treatment of immune and metabolic disorders, as well as cancer. The best developed of these is FTY720, an analogue of sphingoid bases, which has been used in clinical medicine for the treatment of multiple sclerosis. FTY720 is converted to its phosphate derivative by the action of sphingosine kinase 2 (SK2), and in this form, FTY720-Pi modulates immune cell kinetics (primarily the egress of lymphoid cells from lymphoid organs). Myriocin (ISP1), an analogue of sphingosine that functions as a potent inhibitor of serine palmitoyltransferase (SPT), has been evaluated in vivo as a metabolic modulator with favourable effects in diabetes and in metabolic syndrome. Short-chain analogues of ceramide, such as $\mathrm{C} 6$ ceramide, are being formulated in nano-liposomal particles for use in leukaemia.

Many of the enzymes of sphingolipid metabolism have themselves become targets for therapeutics. As mentioned above, SPT has been targeted in animal studies by myriocin. SK1 has been studied extensively, and high-potency (and high-specificity) inhibitors are now available ${ }^{172,173}$. However, clear indications for use have yet to be determined ${ }^{174}$. Inhibitors of acid ceramidase are the subject of intensive development ${ }^{175}$. 
In the case of sphingosine-1-phosphate (S1P), its G protein-coupled receptors are receiving increasing attention as therapeutic targets. Ozanimod, an agonist of S1P receptor 1 (S1PR1) and S1PR5, has shown modest effects in human ulcerative colitis ${ }^{176}$. In addition, a monoclonal antibody against S1P has been developed and has shown moderate responses in a phase II trial in patients with metastatic renal cancer ${ }^{177}$.

Notably, sphingolipids are present not only in mammalian cells but also in certain parasites, including fungi and protozoa. Some of the enzymes involved in their metabolism have no direct human counterparts, and thus they can serve as targets for the development of anti-parasitic drugs ${ }^{178}$.

\section{Compartmentalization of sphingolipids and their metabolism}

An important layer in sphingolipid metabolism is the presence of the multiplicity of enzyme isoforms that are products of distinct genes with specific localization, action and/or substrate specificity. For example, there are at least five distinct ceramidases and five distinct sphingomyelinases that localize to the plasma membrane, endoplasmic reticulum (ER), Golgi, nucleus, lysosome, mitochondria or extracellular space. There are also six different CerSs that show distinct preferences for fatty acids of various lengths (FIG. 1B), thus contributing to the remarkable complexity of ceramides (and their downstream anabolic metabolites). While the CerSs appear to localize primarily to the ER, they have also been specifically noted in the nuclear membrane and in mitochondria.

Given that ceramides, sphingomyelins and some of the glycosphingolipids are hydrophobic (glycosphingolipids with very complex headgroups are much more amphipathic), it is axiomatic that these molecules will reside in the compartments where they are generated (either hydrolytically or synthetically) unless they are specifically transported. Accordingly, specific transporters have been identified for ceramide (ceramide transfer protein (CERT)), S1P (protein spinster homologue 2 (SPNS2)), C1P (C1P transfer protein (CPTP)), and glucosylceramide (phosphatidylinositol-four-phosphate adaptor protein 2 (FAPP2; also known as PH domain-containing family A member 8)) (FIG. 2). Ceramide, as a neutral lipid, appears to readily flip across membrane leaflets, at least in model membranes. This flipping has functional consequences. For example, in the Golgi, ceramide has to flip to serve as the substrate of SMSs in the synthesis of sphingomyelin, as the active site of SMS enzymes faces the lumen of the Golgi ${ }^{1}$. By contrast, synthesis of glucosylceramide from ceramide appears to occur on the cytosolic side of the Golgi. This then necessitates the flipping of glucosylceramide in order to serve as a substrate for the synthesis of higher glycosphingolipids (FIG. 2). The exact machineries involved in these flipping events are not known. In addition to ceramide, evidence points to the existence of a small pool of sphingomyelin on the cytosolic side of the plasma membrane that may serve as substrates for neutral sphingomyelinase 2 (nSMase2) ${ }^{19}$. In freeze-fracture studies, this pool of sphingomyelin appears to be highly clustered ${ }^{20}$, but it is not known how the pool is generated (whether by slow flipping of sphingomyelin from the outer leaflet or by mixing of the inner and external leaflets as may occur during endocytosis and exocytosis). Sphingomyelin is also enriched in a subset of secretory vesicles ${ }^{21}$. 
The highly bioactive S1P undergoes transporter-mediated translocation from inside the cell through the action of SPNS2 (FIG. 2) and several ABC transporters ${ }^{22}$ to the extracellular space, where it can access its S1P receptors. Loss of function of SPNS2 leads to the loss of several actions of S1P that appear to require its flipping to reach its receptors.

It is thus becoming clear that the site of action of bioactive lipids and their metabolism is subjected to targeted regulation by mechanisms controlling their transport and membrane leaflet localization.

\section{Structures of enzymes of sphingolipid metabolism and key transporters}

Our understanding of the molecular and structural underpinnings of sphingolipid metabolism has come from high-resolution crystal structures of key components of these pathways, including $\mathrm{SPT}^{23}$, acid and alkaline sphingomyelinases ${ }^{24-27}$, acid cerebrosidase ${ }^{28}$, sphingosine kinase $1(\mathrm{SK} 1)^{29}$, neutral ceramidase (NCDase $)^{30}$, nSMase, fatty acid ahydroxylase $^{31}$, the adiponectin receptor (which is proposed to act as a ceramidase) ${ }^{32}$ and S1P receptor 1 (S1PR1) ${ }^{33}$, as well as the lipid-transfer proteins CERT (for ceramide) $)^{34}$, CPTP (for C1P) $)^{35}$ and glycolipid transfer protein (GLTP; for lactosylceramide) ${ }^{36}$. Together, these studies have defined the catalytic mechanisms for these enzymes, revealed how they bind and recognize their lipid substrate, aided drug development and allowed us to directly visualize the mechanisms by which sphingolipids can be transported between membranes. A common feature of many of these sphingolipid enzymes and transporters is the presence of a large hydrophobic cavity within the protein structure that is used, at least in part, to extract the target lipid from the membrane.

\section{Mechanisms and downstream targets}

Our understanding of the mechanisms of action of bioactive sphingolipids is rather sparse. S1P has been the most studied bioactive sphingolipid. S1P is mostly secreted from cells and binds to one of five S1PRs, which are G protein-coupled receptors (GPCRs) ${ }^{37}$. These receptors then mediate all known extracellular actions of S1P through canonical GPCR signalling pathways. S1P can also act intracellularly, where it binds to histone deacetylases (HDACs), inhibiting their activity and thereby modulating histone acetylation ${ }^{38}$ (FIG. 3a).

Ceramide has been shown to activate protein phosphatases of the PP1 and PP2A families in vitro and in cells, thereby having important roles in the regulation of the cellular phosphoproteome. Several phosphosubstrates have been defined for this action of ceramide, including serine/threonine protein kinase AKT, protein kinase $\mathrm{C}$ and ezrin ${ }^{39}$ (FIG. 3a). More recently, ceramide-activated phosphatases have been shown to mediate the effects of palmitate on vascular endothelial growth factor signalling, possibly by interfering with activation of endothelial nitric oxide synthase ${ }^{40}$. In addition, ceramide has been shown to regulate the interaction of PP2A with its inhibitor SET (also known as I2PP2A), and this has been implicated in the immunosuppressive functions of regulatory $\mathrm{T}$ cells ${ }^{41}$. A strong case has evolved in yeast for the PP2A homologue Sit4 as a target and mediator of many of the actions of yeast ceramides, including regulation of the cell cycle and mitochondrial function $^{42}$. Additional studies implicate lysosomal ceramide in triggering degradation of Xlinked inhibitor of apoptosis protein (XIAP) in the apoptotic pathway through activation of 
cathepsin $\mathrm{B}^{43}$. In a recent study, diverting ceramide to mitochondria was shown to induce apoptosis ${ }^{44}$, supporting an independent role of the mitochondrial ceramide pool, as noted previously ${ }^{45}$. Unfortunately, there is a paucity of results that illuminate the actions of specific species of ceramides.

The mechanisms of action of sphingosine remain poorly defined in spite of a large number of downstream effects (TABLE 1; Supplementary information S2 (table)) and targets, including protein kinase $\mathrm{C}$. $\mathrm{C} 1 \mathrm{P}$ has been shown to interact with several targets, including cytosolic phospholipase A2 (cPLA2) and acid sphingomyelinase (aSMase), and the existence of cell surface receptors for C1P was suggested, but more detailed mechanistic insights for this and other sphingolipid species are lacking thus far.

\section{Cellular functions}

The plethora of functions now attributed to bioactive sphingolipids is immense and touches almost all major aspects of cell biology, including roles in cell growth, the cell cycle, cell death, cell senescence, inflammation, immune responses, cell adhesion and migration, angiogenesis, nutrient uptake, metabolism, responses to stress stimuli and autophagy (TABLE 1; Supplementary information S2 (table)). The studies of sphingolipid functions have been propelled by the increasing precision of tools such as RNA silencing and, more recently, CRISPR-mediated deletion of specific genes encoding sphingolipid enzymes, as well as by the use of model organisms. There have been several informative reviews on the biological functions of bioactive sphingolipids $1,39,46,47$. Here, we focus on some of the more developed aspects of sphingolipid biology that are supported by multiple lines of investigation and often come from several laboratories.

\section{Insights from yeast studies}

Studies in Saccharomyces cerevisiae have firmly established multiple roles for sphingolipids in the adaptive responses to heat stress, which increases metabolic flux through the de novo pathway, most likely owing to increased uptake of serine. The sequentially generated sphingoid bases, sphingoid base phosphates and ceramides have been implicated in mediating key responses to elevated temperatures, including transient cell cycle arrest, arrest in translation and sequestration of mRNA and regulation of nutrient permeases ${ }^{42,48,49}$. In a combined 'omic' approach, specific ceramides were implicated in specific transcriptional responses, regulating the expression of genes involved in iron transport, acid sensitivity and RNA metabolism, illustrating the complexity of ceramide functions in yeast ${ }^{48}$. Cell cycle checkpoints mediated by mitosis inhibitor protein kinase Swe1 were attributed to ceramides, in particular C18:1 ceramide ${ }^{50,51}$. Additional functions for yeast ceramides include regulation of the diauxic shift and mitochondrial function, the DNA damage response and regulation of responses to reactive oxygen species 42 .

\section{Cell adhesion and migration}

A critical role is emerging for bioactive sphingolipids in the regulation of cell adhesion, migration and invasion. Recent studies implicated SK1 and SK2 (the enzyme producing $\mathrm{S} 1 \mathrm{P})$ in filopodia formation, cell migration and invasion in response to different stimuli, 
including epidermal growth factor, in a mechanism that involves induction of phosphorylation of the ezrin, radixin and moesin (ERM) family protein ezrin ${ }^{52}$, which is known for its function in regulating cellular motility ${ }^{53}$. The mechanism involves the activation of S1PR2 and implicates the transporting functions of SPNS2 (REF. 52). Remarkably, a recent study using a genome-wide in vivo screen in mice identified SPNS2 as a prominent promoter of metastasis ${ }^{54}$. Also of interest, $S 1$ pr 2 -knockout mice are deaf ${ }^{55}$, similarly to mice deficient for another ERM protein, $\operatorname{radixin}^{56}$, as well as to Spns2-knockout mice ${ }^{57}$, suggesting a common pathway involving these three components. Reciprocally, ezrin can be dephosphorylated in a mechanism involving ceramide-mediated activation of the protein phosphatase PP1 $a^{58}$. These studies illustrate the mechanistic and functional crosstalk between bioactive sphingolipids (FIG. 3a).

\section{Formation of membrane domains, exosomes and endosomes}

Sphingolipid metabolism has been shown to affect membrane organization and dynamics, as well as vesicular transport (FIG. 3b).

Substantial literature supports the existence of specialized membrane domains, especially in the plasma membrane, which are enriched in specific lipid species, including sphingolipids (with an important contribution of ceramides). Although the precise nature and function of these domains, which also include rafts, are still not clear, they could have important roles in regulating the function of membrane receptors. Thus, ceramides could influence cell signalling by contributing to (and potentially promoting) the formation of membrane domains ${ }^{59}$.

Suggesting the role of sphingolipids in membrane dynamics and signalling more broadly, a landmark study identified a role for nSMase and the resultant ceramide in the formation and secretion of exosomes, a specific subtype of secreted vesicles that have been implicated in cell-cell signalling and communication ${ }^{60}$. Subsequently, several studies have disclosed that inhibition of nSMase 2 can interfere with the cargo composition of these vesicles, including microRNA (miRNA $)^{61}$, prion proteins ${ }^{62}$ and other cargo proteins with important roles in neurodevelopment and neurodegeneration. However, inhibition of SMS2 promotes exosome secretion by neurons and could be linked to the clearance of the neurotoxic amyloid- $\beta$ by microglia ${ }^{63}$, suggesting therapeutic possibilities in Alzheimer disease by modulating the metabolism of sphingolipids.

Furthermore, SK1 was implicated in endosome regulation (FIG. 3b), whereby knockdown of SK1 led to defects in endocytic recycling, suggesting an important role for S1P in endocytic membrane trafficking that is likely independent of its role in signalling through S1PRs ${ }^{64}$. Sphingolipids also appear to be involved in the internalization of viruses, as illustrated by the implication of SMS2 in the fusion of the HIV viral envelope with the host cell plasma membrane ${ }^{65}$.

It was also demonstrated that a specific species of sphingomyelin, C18 sphingomyelin, is recognized by $\mathrm{p} 24$, a component of the coatomer protein COPI comprising secretion machinery, and this interaction is important for vesicular trafficking ${ }^{66}$. This example 
highlights the importance of the distinct cellular roles of molecular subspecies of sphingolipids mentioned above.

Finally, the existence of an intricate crosstalk between sphingolipid metabolism and membrane dynamics has been illustrated by cellular studies, demonstrating that a finely tuned mechanism involving the combined action of CERT, SMSs and protein kinase D regulates both sphingomyelin levels and vesicular trafficking from the Golgi ${ }^{67}$.

\section{Implications in DNA damage response}

Early studies had demonstrated that many cytotoxic and DNA-damaging agents induced profound changes in the levels of sphingolipids, in particular leading to increased levels of ceramide. Accordingly, enzyme-centric studies identified SK1, alkaline ceramidase 2 (ACER2), nSMase2 and CerSs as specific players in the DNA damage response (DDR) (see also BOXES 1,2). SK1 protein levels are subjected to p53-induced degradation, and this has been implicated in regulating cell survival and inflammatory responses. This connection was supported by an in vivo study demonstrating that $S k 1$ (also known as $S p h k 1$ ) knockout protected mice from thymic lymphomas induced by the loss of p53 (REF. 68). By contrast, ACER 2 mRNA levels are induced by $\mathrm{p} 53$, and this protects cells with wild-type p53 from ceramide-mediated cell death ${ }^{69}$. Similarly, nSMase2 is induced during the DDR and appears to have a role in cell cycle regulation ${ }^{70}$. An analogous role has been shown for the $S$. cerevisiae protein Isc1 (a homologue of nSMase 2$)^{50}$. Additional studies have implicated CerSs as mediators of cytotoxic responses of cancer cells to various chemo therapeutic and stress agents (reviewed in REF. 11; see also BOX 1).

\section{Roles in senescence, ageing, autophagy and apoptosis}

In yeast, lag1 and lac1 were the first genes identified as important determinants of lifespan. Eventually, it was determined that the products of these genes function as CerSs ${ }^{71}$. In parallel, mammalian cell studies had implicated ceramide as a key regulator of cell senescence ${ }^{5}$. More recently, the three CerSs in Caenorhabditis elegans were demonstrated to regulate lifespan, interestingly in both directions depending on the enzyme. This study highlights the complexities of sphingolipid metabolism, showing that the variation in ceramide composition determined by the activity of the different CerSs can greatly affect animal lifespan; these different effects were further linked to the modulation of the response to dietary restriction and activation of autophagy ${ }^{72}$. Along similar lines, haplotype analysis of human longevity-associated genes identified $C E R S 1$ as a key gene enhancing exceptional survival (those living into the 10th decade) ${ }^{73}$.

Autophagy has recently emerged as a highly regulated process in which sphingolipids are importantly involved. Ceramides regulate both cell survival- and cell death-linked autophagy by regulating nutrient transporters, ER stress and mitophagy (reviewed in REF. 74). Accumulation of dihydroceramide by inhibiting the desaturation of ceramides (see BOX 2) has been implicated in regulating autophagy ${ }^{75}$, in particular autophagy-induced death of cancer cells ${ }^{76}$.

Another highly regulated process in which bioactive sphingolipids have emerged as key players is cell death. Ceramide has been identified as a key regulator of apoptosis ${ }^{77}$, and this 
cellular activity of ceramide has since been extensively studied (reviewed in REF. 39) (FIG. 3a). Of note, several enzymes that regulate the generation of ceramides, including CerSs, SMases, SK1 and SK2, are distributed in different cellular compartments such as mitochondria, the plasma membrane or lysosomes (Supplementary information S3 (table)), which can influence distinct mechanisms of cell death (apoptosis versus necroptosis) depending on the activity of these enzymes ${ }^{44,78}$.

\section{Roles in (patho)physiology}

An increasing appreciation of the roles of sphingolipids in organismal physiology and pathophysiology is driven by the translation of basic cell studies to animal and human processes, which in turn has been greatly facilitated by the development of knockout mice for a majority of enzymes of sphingolipid metabolism (Supplementary information S3 (table)). Equally as gratifying has been the appreciation of critical roles of sphingolipids from studies beyond the sphingolipid domain (for example, an unbiased screen uncovered the role of SPNS2 in metastasis ${ }^{54}$ ). Here, we discuss a few best-studied examples of how sphingolipids affect organismal biology.

\section{Immune and inflammatory functions}

A strong case for S1P in the function of the immune system has been established ${ }^{46}$ (FIG. 3c). This has found a direct application in the treatment of multiple sclerosis with FTY720 (fingolimod), a sphingoid base analogue ${ }^{79}$. Briefly, $\mathrm{T}$ cell egress is controlled by a gradient of S1P between the lymph and plasma ${ }^{80}$ that is established by the secretion of S1P from haematopoietic and endothelial cells into plasma ${ }^{81}$. S1PR1 expressed on lymphocytes then responds to this gradient, leading to the migration of lymphocytes from lymphoid tissues into the circulation ${ }^{82}$. This process is inhibited by FTY720, which is converted by SK2 into FTY720 phosphate, which then acts to tonically suppress S1PR1, thus leading to the sequestration of $\mathrm{T}$ cells in the thymus and lymph nodes. Consistent with these findings, phospholipid phosphatase 3 (LPP3), an S1P phosphatase that hydrolyses S1P, was found to promote lymphocyte egress, presumably by decreasing S1P levels in target organs and thus maintaining a blood (high)- tissue (low) S1P gradient ${ }^{83}$. In addition, a specific pool of S1P bound to high-density lipoprotein (HDL) has been shown to regulate lymphopoiesis and neuroinflammation not only by regulating delivery of S1P to the blood but also by modulating its receptor signalling pathways ${ }^{84}$.

A connection has also emerged between bioactive sphingolipids and the cPLA2cyclooxygenase 2 (COX2) pathway, which generates bioactive prostaglandins. Studies have implicated SK1 and S1P in regulating the expression of COX2, although the mechanisms remain unclear. Additionally, $\mathrm{C} 1 \mathrm{P}$ and the enzyme that forms it, ceramide kinase, have been implicated in the direct activation of cPLA2 in response to cytokines ${ }^{85}$. These results suggest an important role for $\mathrm{C} 1 \mathrm{P}$ and $\mathrm{S} 1 \mathrm{P}$ in inflammatory responses. However, it was shown that deletion of SK1 from macrophages is not required for inflammatory responses but did sensitize these cells to autophagy-induced death ${ }^{86}$.

Ongoing studies also suggest roles for aSMase in sustaining inflammation through the production of inflammatory cytokines, especially interleukin-6 (IL-6) and CC-chemokine 
ligand 5 (CCL5) in response to the action of tumour necrosis factor (TNF) and IL-1 (REF. 87). Clinically, levels of serum aSMase have been shown to predict mortality in patients in intensive care units at risk of developing systemic inflammation ${ }^{88}$, an effect most likely related to the induction of aSMase release by TNF.

\section{Multilevel involvement of sphingolipids in cancer cell biology}

Bioactive sphingolipids have emerged as key regulators of several, if not most, of the key attributes of cancer and cancer cell biology (Supplementary information S3 (table)). On the one hand, S1P has been implicated in tumour cell survival, cancer inflammatory pathways, angiogenesis, resistance to chemotherapy and cancer cell invasion. On the other hand, ceramides have been implicated in cancer cell apoptosis, growth arrest, mediating sensitivity to chemotherapeutics and senescence ${ }^{89,90}$. Here, we discuss some of the more mature findings implicating sphingolipids in specific cancers.

A strong case has evolved for a role for SK1 in colon cancer, especially colitis-associated cancer (CAC). These studies were prompted by the finding that SK1 is involved in the regulation of COX2 and cell inflammatory responses ${ }^{91}$. Human colon cancer showed upregulation of SK1 in 89\% of cases, and this correlated with the high levels of COX2. Higher levels of SK1 in colon cancer in an Asian population correlated with advanced stage and predicted increased mortality ${ }^{92}$. $S k 1$-knockout mice were partly resistant to chemically induced colitis, and they showed significant attenuation of colon cancer in a model of $\mathrm{CAC}^{93}$. Mechanistic studies defined a role for SK1 in mediating the induction of COX2 in vivo ${ }^{93}$ as well as in the regulation of the expression of signal transducer and activator of transcription 3 (STAT3), which is a key transducer of pro-inflammatory signals from several types of plasma membrane receptors ${ }^{94}$. Moreover, loss of SK1 also decreased the growth of adenomas in a mouse model of intestinal cancer ${ }^{95}$. In addition to the increase in SK1, S1P lyase is downregulated in colon cancer tissues, further promoting S1P signalling ${ }^{96}$. Interestingly, knockout of $S K 2$ resulted in reciprocal increases in SK1, which were implicated in increased risk of colon cancer in mice ${ }^{94}$. In line with the studies presented above, targeting SK1 with inhibitors showed activity against colon cancer in xenografts ${ }^{97}$ and CAC mouse models ${ }^{98}$. Thus, these studies confirm that the S1P pathway contributes to colon carcinogenesis, likely by regulating synthesis of prostaglandins and other inflammatory mediators.

Interestingly, and in contrast to S1P, ceramide levels have been shown to be reduced in human colon cancer, consistent with the overall opposing functions of these two lipid mediators (FIG. 1A). As such, deletion of NCDase, which augments ceramide levels, protected mice from the development of colon cancer in a carcinogen-induced model of colon cancer ${ }^{99}$. However, it should be noted that the involvement of NCDase appears to be distinct from the role of SK1 in CAC.

The SK1-S1P pathway has also been implicated in several other cancers, including glioblastoma, head and neck cancer, renal cancer and several leukaemias ${ }^{100}$. Notably, the cancer-promoting roles of this pathway extend beyond actions on cancer cells (such as protection from apoptosis). For example, S1P has emerged as a key regulator of angiogenesis with widely important implications in vascular biology and cancer ${ }^{100-102}$, and 
SK1 is induced in many cancers in response to hypoxia and in renal cell carcinoma with mutations in the von Hippel-Lindau (VHL) gene. The resultant paracrine increases in S1P in hypoxia have been implicated in angiogenesis and lymphangiogenesis ${ }^{103}$.

Acid ceramidase (ACDase) is another enzyme that has received considerable attention in the context of cancer biology, in particular in the development of therapeutics ${ }^{104,105}$. The levels of this enzyme are increased in several cancers, including head and neck cancer, melanoma and prostate cancer, and correlate with the advanced pathology of these tumour types.

ACDase serves to attenuate levels of ceramide, and inhibitors of ACDase sensitize cancer cells to chemotherapy and radiotherapy in cell culture and in xenografts ${ }^{104}$. A newly developed and potent inhibitor of ACDase synergized with several chemotherapeutics to induce melanoma cell death ${ }^{106}$. Higher levels of ACDase correlate with increased aggressiveness of melanoma, whereas the expression of aSMase, which promotes ceramide generation, is inversely correlated with tumour stage ${ }^{107}$, indicating a strong correlation between low levels of ceramide and advanced stages of disease in many cancers. However, evaluation of the expression of ACDase in breast cancer showed that it is expressed at higher levels in low-risk, luminal A type breast cancer, and high expression was associated with better prognosis ${ }^{108}$. Therefore, whereas ACDase appears to, at least in part, drive malignancy in head and neck cancer, melanoma and prostate cancer, it has rather protective functions in breast cancer.

As another example, platelet-derived aSMase was implicated in promoting cell adhesion and metastasis of melanoma ${ }^{109}$. In addition, aSMase in endothelial cells has been proposed to have a key role in tumour angiogenesis, as it has been shown to mediate sensitivity to antiangiogenic therapy by elevating the levels of ceramide ${ }^{110}$.

Exciting studies are also pointing towards the usability of sphingolipid levels and the activity of their metabolic enzymes as biomarkers in cancer. A recent metabolomic profiling of pancreatic adenocarcinoma identified changes in sphingolipid levels, including elevation of sphingomyelin, as a dominant component and determinant of a 'lipogenic' subtype defined by the investigators as showing distinct metabolism of lipids ${ }^{111}$. Increases in plasma ceramide levels have been shown to be predictive of the response of metastatic tumours to radiation therapy ${ }^{12}$. Along similar lines, CerS6 emerged as a strong predictor of survival in human colon cancer ${ }^{113}$.

These results indicate that specific perturbations in one or more pathways of bioactive sphingolipids can affect various key components of cancer development (for example, growth, migration, angiogenesis and immune signalling) and responses to therapies. These perturbations are emerging as cancer-specific, possibly due to the distinct functions of various enzymes in different tissues and under distinct conditions. Thus, these roles need to be appreciated in this tissue-specific context.

\section{Metabolic functions and connections to diabetes and the cardiovascular system}

A strong case is emerging for a role for ceramides in regulating metabolic functions, and changes in levels of ceramide species have been implicated in various metabolic syndromes, such as fatty liver disease ${ }^{114}$, obesity ${ }^{115}$ and insulin resistance ${ }^{47,116}$. Cell and in vivo studies 
have demonstrated that increased ceramide levels result in attenuation of insulin action, most likely via inhibition of AKT (FIG. 3a). This inhibitory pathway appears to be particularly engaged by the increased supply of palmitic acid, which can drive ceramide synthesis directly as a precursor substrate. Increased ceramide synthesis in the context of insulin resistance was also shown to be driven by inflammatory agents (for example, agonists of Toll-like receptors, TNF and interleukins) ${ }^{47}$. Attenuation of ceramide generation in vivo, by use of inhibitors of SPT or dihydroceramide desaturase or mice deficient in these enzymes, improves insulin signalling and glucose and lipid metabolism in diabetes and alleviates obesity-associated metabolic changes and cardiac dysfunction ${ }^{117,118}$. Furthermore, mice deficient in CerS6 are protected from weight gain and glucose intolerance ${ }^{119}$ induced by a high-fat diet, and overexpression of ACDase led to a reduction of hepatic steatosis ${ }^{120}$. Similarly to ceramide, other sphingolipids, including sphingomyelin and glucosylceramide, have also been shown to inhibit insulin action ${ }^{121}$. Accordingly, Sgms2-knockout mice, in which sphingomyelin levels are reduced, showed increased sensitivity to insulin and resistance to obesity induced by a high-fat $\operatorname{diet}^{122}$. Notably, these effects were observed despite an associated increase in the levels of ceramide, suggesting that ceramide is not the primary mediator of insulin signalling inhibition or that in the context of Sgms 2 knockout, the elevated ceramide is diverted to other pathways. Interestingly, different metabolic functions were found for $S G M S 1$, deletion of which caused decreased insulin secretion and induced oxidative stress in white adipose tissue, leading to the destruction and dysfunction of white adipocytes ${ }^{123}$.

Metabolic roles of S1P are also emerging. In mice, deletion of the gene encoding S1P phosphatase 2 (Sgpp2), which degrades S1P, was associated with basal ER stress in pancreatic $\beta$-cells and increased sensitivity to a high-fat diet ${ }^{124}$. Consistent with these results, deletion of the gene encoding SK1 was shown to protect against diet-induced hepatic steatosis $^{125}$. In support of the role of ER stress in mediating these metabolic effects of S1P, cell-based studies have demonstrated involvement of S1P signalling in regulating heat shock proteins, the unfolded protein response in the ER and ER stress ${ }^{126}$.

Sphingolipids, and especially ceramide and sphingosine, have been implicated in vascular disease, including atherosclerosis and ischaemic injury. Increased activity of aSMase and levels of ceramides have been linked to atherogenesis; the mechanism possibly involves secreted aSMase that may act on lipoprotein-associated sphingomyelin to convert it to ceramide $^{127}$. Accordingly, attenuation of aSMase was demonstrated to act protectively against retinal ischaemic injury in mice ${ }^{128}$. In support of the general impact of sphingolipid metabolism on vasculature, inhibition of de novo synthesis of sphingolipids with myriocin attenuated myocardial reperfusion injury ${ }^{129}$.

From a clinical perspective, a very compelling case is developing for the utility of measuring serum sphingolipids (mostly ceramides) as indicators of metabolic disorders and atherosclerotic cardiovascular diseases. For example, plasma levels of C24 and C26 ceramides as well as deoxy-C24 ceramide have been associated with diabetic neuropathy ${ }^{130}$. Elevated C18:0 and C18:1 ceramides were also shown to be strong predictors of major adverse cardiovascular effects in healthy subjects ${ }^{131}$. Similarly, C18:1 ceramide was found to be a key predictor of high amounts of necrosis after coronary angiography procedures ${ }^{132}$. 
Another study identified C24:1 ceramide and sphingomyelin as being strongly associated with cardiovascular mortality ${ }^{133}$. Higher levels of plasma C22:0 and C24:0 ceramides have also been shown to predict less improvement in verbal memory in response to exercise in patients with coronary artery disease in which these skills decline ${ }^{134}$. In addition to the use of ceramides as biomarkers, serum S1P levels inversely correlated with atherosclerotic disease ${ }^{135}$, whereas deoxysphingolipids have been shown to serve as a biomarker in diabetes progression $^{136}$.

\section{Sphingolipids as drivers of neurodevelopment and neurodegeneration}

Genetic defects in various enzymes of sphingolipid metabolism reveal important roles for these enzymes in brain development and health (reviewed in REF. 6). These compelling conclusions have come from converging studies using both mouse knockouts and genetic tracing of human disease. These include defects in fatty acid 2-hydroxylase (FA2H) ${ }^{137}$, ACER3 (REF. 138), SPTSSB (a small subunit of SPT) ${ }^{139}$, CerS1 (REF. 140) and CerS2 (REFS 141,142) (Supplementary information S3 (table)). These recent studies suggest that key sphingolipid metabolites are critical for normal brain development and function. In most cases, the sphingolipid species that may cause neural dysfunction are not known. However, in a recent study of Cers 1 knockout, neuronal production of C18:1 ceramides and other sphingolipids was found to be impaired, leading to the accumulation of sphingoid bases and neurodegeneration. These effects could be corrected by overexpression of CerS2 (REF. 143), suggesting that sphingoid bases are the candidate mediators of neuronal injury.

In addition, deficiency of nSMase 2 was shown to decrease pathology associated with Alzheimer disease in mice ${ }^{144}$, whereas deletion of Asah2, which encodes NCDase, attenuated brain damage associated with traumatic brain injury ${ }^{145}$.

Taken together, these studies begin to define several causal roles for sphingolipids in normal brain functions and as mediators of neuronal damage and neurodegenerative disorders.

\section{Skin-specific sphingolipids and their functions}

Skin is characterized by the presence of unusual sphingolipids based on ultra-long fatty acyl chains as well as fatty acyl groups linked on the $\omega$-end of the $N$-linked fatty acids (thereby generating a three-chain rather than a two-chain molecule). Loss of function of several enzymes involved in sphingolipid metabolism leads to various forms of skin and hair defects that are often fatal - loss of CerS3 (REF. 146), elongation of very-long-chain fatty acids protein 4 (ELOVL4), CYP4F22 (the $\omega$-hydroxylase) ${ }^{147}$, glucosylceramide synthase (UGCG) ${ }^{148}$ or PNPLA1 (REF. 13) leads to defects in skin epithelium organization and function, whereas loss of ELOVL3 and ELOVL4 (REFS 149,150), CerS4 (REF. 151) or ACER1 (REF. 152) results in hair loss phenotypes. At least for some of these enzymes, the defects resulting from their deficiency appear to be mechanical in nature, in that the skin fails to form an impermeable barrier owing to the loss of its specialized, long-chain ceramides. 


\section{Tissue-specific sphingolipid metabolism in bone and cartilage development}

A strong case has been made for a role for nSMase 2 in bone and cartilage development.

Knockout of nSMase 2 resulted in a stunted growth phenotype of the mice ${ }^{153}$. Independently, it was shown that frolfro mice, a model of osteogenesis imperfecta, carry an inactivating truncation in Smpd3 (encoding nSMase2). Subsequent studies have shown that the defects in development in bone and cartilage are likely due to tissue-autonomous and/or cellautonomous actions of nSMase 2 (REF. 154). Accordingly, in chondrocytes, nSMase 2 was demonstrated to be a downstream target for bone morphogenetic protein 2 (REF. 155) and for all-trans retinoic acid (ATRA) ${ }^{156,157}$, key regulators of bone and cartilage development.

\section{Conclusions and perspectives}

The extent to which mammalian cells have evolved the network of bioactive sphingolipids to control many critical cellular and organismal functions is remarkable. The complexity of this network makes sphingolipids extremely difficult to study, particularly in the context of the entire organism, where specific functions in individual tissues and organs crosstalk and converge. Much has been accomplished in terms of defining the basic biochemical components and tools for studying and modulating pathways in this network and in advancing our understanding of the multifaceted roles of sphingolipid species. However, much remains to be achieved. In particular, molecular and biochemical mechanisms that regulate the many enzymes of sphingolipid metabolism and the flux through the system, with its many nodes and interconnections and compartments, require further investigation and refining. Equally important, there is a compelling need to understand the mechanisms of actions of the many ceramide species and sphingoid bases, as well as other emerging bioactive sphingolipids. Only such a level of understanding, considering individual sphingolipid species and their changes in different physiological and pathological contexts, will allow a precise delineation of the specific roles and functions of these pathways and how they fit in the larger universe of cell regulation and biology.

\section{Supplementary Material}

Refer to Web version on PubMed Central for supplementary material.

\section{Acknowledgments}

The authors thank the members of their laboratories and M. Airola, C. Luberto, C. Clarke, D. Canals, C. Senkal, C. Rhein and F. Velazquez for helpful discussions. The authors are very grateful for the contribution of M. Hernandez for assembling table 1 and supplementary information tables S2 and S3. Due to space limitations, the authors have striven to reference the more recent studies pertinent to the presentation while directing the readers to more in-depth targeted reviews. The authors apologize for the multitude of sphingolipid investigators whose works they could not cite in this Review.

\section{Glossary}

\section{Prostaglandins}

Bioactive, acidic lipids with various hormone-like activities, including modulation of inflammation, regulation of blood flow and blood pressure and reproduction. 


\section{Gangliosides}

A major subtype of sphingolipids composed of ceramide and an oligosaccharide that contains at least one sialic acid residue.

\section{$\omega$-Acylation}

Acylation of fatty acids at the $\omega$-position (last position) in the acyl chain.

\section{Freeze-fracture studies}

A form of electron microscopy involving freezing in order to preserve lipid membrane structures.

\section{G protein-coupled receptors (GPCRs)}

Heptahelical membrane receptors that bind and regulate $\mathrm{G}$ proteins.

\section{Globosides}

A subtype of sphingolipids with a ceramide associated with at least two sugars, but no sialic acid.

\section{Diauxic shift}

The shift in growth from rapid fermentative to aerobic glycolysis.

\section{Microglia}

A type of neural cell arising from macrophages or their precursors that serves supportive and protective functions in the central nervous system.

\section{ER stress}

Endoplasmic reticulum (ER) dysfunction due to stress stimuli that result in increased accumulation of misfolded proteins in the ER.

\section{Necroptosis}

A regulated form of necrotic cell death associated with immune and inflammatory responses.

\section{Multiple sclerosis}

A degenerative disease of the nervous system associated with a loss of myelination (covering) of axonal sheaths.

\section{T cell egress}

Lymphocyte migration from the thymus and lymph nodes into the bloodstream.

\section{von Hippel-Lindau ( $V H L)$ gene}

A gene whose mutations can result in von Hippel-Lindau disease. It encodes a protein that participates in the regulation of the levels of hypoxia-inducible factor (HIF) through degradation.

\section{Luminal A type breast cancer}

A subtype of breast cancer in which the cells appear to resemble most cells of the luminal lining of the breast ducts. 
Insulin resistance

A state in which cells, tissues or organisms fail to respond normally to insulin.

\section{Glucose intolerance}

Also known as impaired glucose tolerance. A pre-diabetic state involving hyperglycaemia and usually poor responsiveness to insulin.

\section{Hepatic steatosis}

Condition associated with non-alcoholic fatty liver disease with increased accumulation of fat in liver cells, usually in the form of triglycerides.

\section{Ischaemic injury}

Tissue and cell injury that results from a decrease in or interruption of the blood supply.

\section{Reperfusion injury}

Injury or damage to tissues resulting from the reoxygenation of previously ischaemic tissues.

\section{Diabetic neuropathy}

Dysfunction of the peripheral and autonomic nervous system that arises from long-standing diabetes.

\section{Coronary angiography}

An invasive procedure using dyes in the bloodstream to visualize the coronary circulation using radiography.

\section{Osteogenesis imperfecta}

A group of genetic disorders characterized by brittle bones.

\section{References}

1. Hannun YA, Obeid LM. Principles of bioactive lipid signalling: lessons from sphingolipids. Nat Rev Mol Cell Biol. 2008; 9:139-150. [PubMed: 18216770]

2. Thudichum, JLW. A Treatise on the Chemical Constitution of the Brain. Archon Books; 1962. This is the first documented isolation of the sphingolipids, and includes the coining of the term 'sphingosin'

3. Hannun YA, Obeid LM. Many ceramides. J Biol Chem. 2011; 286:27855-27862. This review advances the hypothesis that ceramides are indeed a family of distinct molecular species that are products of distinct metabolic enzymes and that the different ceramides may have distinct functions. [PubMed: 21693702]

4. Schulze H, Sandhoff K. Sphingolipids and lysosomal pathologies. Biochim Biophys Acta. 2014; 1841:799-810. [PubMed: 24184515]

5. Huang X, Withers BR, Dickson RC. Sphingolipids and lifespan regulation. Biochim Biophys Acta. 2014; 1841:657-664. [PubMed: 23954556]

6. Astudillo L, et al. Human genetic disorders of sphingolipid biosynthesis. J Inherit Metab Dis. 2015; 38:65-76. This is a comprehensive presentation of the various genetic disorders that are directly caused by defects in sphingolipid metabolism. [PubMed: 25141825]

7. Bode $\mathrm{H}$, et al. HSAN1 mutations in serine palmitoyltransferase reveal a close structure-functionphenotype relationship. Hum Mol Genet. 2016; 25:853-865. [PubMed: 26681808]

8. Hornemann T, et al. The SPTLC3 subunit of serine palmitoyltransferase generates short chain sphingoid bases. J Biol Chem. 2009; 284:26322-26330. [PubMed: 19648650] 
9. Harmon JM, et al. Topological and functional characterization of the ssSPTs, small activating subunits of serine palmitoyltransferase. J Biol Chem. 2013; 288:10144-10153. [PubMed: 23426370]

10. Cingolani F, Futerman AH, Casas J. Ceramide synthases in biomedical research. Chem Phys Lipids. 2016; 197:25-32. [PubMed: 26248326]

11. Wegner MS, Schiffmann S, Parnham MJ, Geisslinger G, Grosch S. The enigma of ceramide synthase regulation in mammalian cells. Prog Lipid Res. 2016; 63:93-119. This is a comprehensive presentation of the functions and regulation of the family of CerSs. [PubMed: 27180613]

12. Sassa T, Kihara A. Metabolism of very long-chain Fatty acids: genes and pathophysiology. Biomol Ther. 2014; 22:83-92.

13. Grond S, et al. PNPLA1 deficiency in mice and humans leads to a defect in the synthesis of omega$O$-acylceramides. J Invest Dermatol. 2017; 137:394-402. [PubMed: 27751867]

14. Senkal CE, et al. Ceramide is metabolized to acylceramide and stored in lipid droplets. Cell Metab. 2017; 25:686-697. This study describes a novel pathway by which ceramide can be diverted or stored as O-acyl-ceramide in lipid droplets. [PubMed: 28273483]

15. Ferreira NS, et al. Regulation of very-long acyl chain ceramide synthesis by acyl-CoA binding protein. J Biol Chem. 2017; 292:7588-7597. [PubMed: 28320857]

16. Wakashima T, Abe K, Kihara A. Dual functions of the trans-2-enoyl-CoA reductase TER in the sphingosine 1-phosphate metabolic pathway and in fatty acid elongation. J Biol Chem. 2014; 289:24736-24748. This study identifies a key enzyme involved in the metabolism and recycling of fatty aldehydes after their generation from the breakdown of S1P. [PubMed: 25049234]

17. Cabukusta B, et al. ER residency of the ceramide phosphoethanolamine synthase SMSr relies on homotypic oligomerization mediated by its SAM domain. Sci Rep. 2017; 7:41290. [PubMed: 28120887]

18. Rajagopalan V, et al. Critical determinants of mitochondria-associated neutral sphingomyelinase (MA-nSMase) for mitochondrial localization. Biochim Biophys Acta. 2015; 1850:628-639. [PubMed: 25484313]

19. Murate M, et al. Transbilayer distribution of lipids at nano scale. J Cell Sci. 2015; 128:1627-1638. [PubMed: 25673880]

20. Abe M, Kobayashi T. Imaging local sphingomyelin-rich domains in the plasma membrane using specific probes and advanced microscopy. Biochim Biophys Acta. 2014; 1841:720-726. [PubMed: 23860017]

21. Deng Y, Rivera-Molina FE, Toomre DK, Burd CG. Sphingomyelin is sorted at the trans Golgi network into a distinct class of secretory vesicle. Proc Natl Acad Sci USA. 2016; 113:6677-6682. [PubMed: 27247384]

22. Nagahashi M, et al. Sphingosine-1-phosphate transporters as targets for cancer therapy. BioMed Res Int. 2014; 2014:651727. [PubMed: 25133174]

23. Wadsworth JM, et al. The chemical basis of serine palmitoyltransferase inhibition by myriocin. $\mathbf{J}$ Am Chem Soc. 2013; 135:14276-14285. [PubMed: 23957439]

24. Zhou YF, et al. Human acid sphingomyelinase structures provide insight to molecular basis of Niemann-Pick disease. Nat Commun. 2016; 7:13082. [PubMed: 27725636]

25. Gorelik A, Illes K, Heinz LX, Superti-Furga G, Nagar B. Crystal structure of mammalian acid sphingomyelinase. Nat Commun. 2016; 7:12196. [PubMed: 27435900]

26. Xiong ZJ, Huang J, Poda G, Pomes R, Prive GG. Structure of human acid sphingomyelinase reveals the role of the saposin domain in activating substrate hydrolysis. J Mol Biol. 2016; 428:3026-3042. [PubMed: 27349982]

27. Gorelik A, Liu F, Illes K, Nagar B. Crystal structure of the human alkaline sphingomyelinase provides insights into substrate recognition. J Biol Chem. 2017; 292:7087-7094. [PubMed: 28292932]

28. Dvir H, et al. X-Ray structure of human acid- $\beta$-glucosidase, the defective enzyme in Gaucher disease. EMBO Rep. 2003; 4:704-709. [PubMed: 12792654]

29. Wang Z, et al. Molecular basis of sphingosine kinase 1 substrate recognition and catalysis. Structure. 2013; 21:798-809. [PubMed: 23602659] 
30. Airola MV, et al. Structural basis for ceramide recognition and hydrolysis by human neutral ceramidase. Structure. 2015; 23:1482-1491. [PubMed: 26190575]

31. Zhu G, Koszelak-Rosenblum M, Connelly SM, Dumont ME, Malkowski MG. The crystal structure of an integral membrane fatty acid a-hydroxylase. J Biol Chem. 2015; 290:29820-29833. [PubMed: 26515067]

32. Vasiliauskaite-Brooks I, et al. Structural insights into adiponectin receptors suggest ceramidase activity. Nature. 2017; 544:120-123. [PubMed: 28329765]

33. Hanson MA, et al. Crystal structure of a lipid G protein-coupled receptor. Science. 2012; 335:851855. This study describes the crystal structure of S1PR1. [PubMed: 22344443]

34. Kudo N, et al. Crystal structures of the CERT START domain with inhibitors provide insights into the mechanism of ceramide transfer. J Mol Biol. 2010; 396:245-251. [PubMed: 20036255]

35. Simanshu DK, et al. Non-vesicular trafficking by a ceramide-1-phosphate transfer protein regulates eicosanoids. Nature. 2013; 500:463-467. This study describes the crystal structure of the C1P transporter. [PubMed: 23863933]

36. Samygina VR, et al. Enhanced selectivity for sulfatide by engineered human glycolipid transfer protein. Structure. 2011; 19:1644-1654. [PubMed: 22078563]

37. Sanchez T, Hla T. Structural and functional characteristics of S1P receptors. J Cell Biochem. 2004; 92:913-922. [PubMed: 15258915]

38. Hait NC, et al. Regulation of histone acetylation in the nucleus by sphingosine-1-phosphate. Science. 2009; 325:1254-1257. This study identifies HDACs as direct nuclear targets of S1P. [PubMed: 19729656]

39. Galadari S, Rahman A, Pallichankandy S, Thayyullathil F. Tumor suppressive functions of ceramide: evidence and mechanisms. Apoptosis. 2015; 20:689-711. [PubMed: 25702155]

40. Mehra VC, et al. Ceramide-activated phosphatase mediates fatty acid-induced endothelial VEGF resistance and impaired angiogenesis. Am J Pathol. 2014; 184:1562-1576. [PubMed: 24606881]

41. Apostolidis SA, et al. Phosphatase PP2A is requisite for the function of regulatory T cells. Nat Immunol. 2016; 17:556-564. [PubMed: 26974206]

42. Teixeira V, Costa V. Unraveling the role of the target of rapamycin signaling in sphingolipid metabolism. Prog Lipid Res. 2016; 61:109-133. This is a comprehensive review of sphingolipid metabolism, function and regulation in yeast. [PubMed: 26703187]

43. Taniguchi M, et al. Lysosomal ceramide generated by acid sphingomyelinase triggers cytosolic cathepsin B-mediated degradation of X-linked inhibitor of apoptosis protein in natural killer/T lymphoma cell apoptosis. Cell Death Dis. 2015; 6:e1717. [PubMed: 25855965]

44. Jain A, Beutel O, Ebell K, Korneev S, Holthuis JC. Diverting CERT-mediated ceramide transport to mitochondria triggers Bax-dependent apoptosis. J Cell Sci. 2017; 130:360-371. [PubMed: 27888218]

45. Birbes $\mathrm{H}$, et al. A mitochondrial pool of sphingomyelin is involved in TNFa-induced Bax translocation to mitochondria. Biochem J. 2005; 386:445-451. [PubMed: 15516208]

46. Spiegel S, Milstien S. The outs and the ins of sphingosine-1-phosphate in immunity. Nat Rev Immunol. 2011; 11:403-415. [PubMed: 21546914]

47. Chaurasia B, Summers SA. Ceramides - lipotoxic inducers of metabolic disorders. Trends Endocrinol Metab. 2015; 26:538-550. [PubMed: 26412155]

48. Montefusco DJ, Matmati N, Hannun YA. The yeast sphingolipid signaling landscape. Chem Phys Lipids. 2014; 177:26-40. [PubMed: 24220500]

49. Epstein S, Riezman H. Sphingolipid signaling in yeast: potential implications for understanding disease. Front Biosci. 2013; 5:97-108.

50. Matmati N, et al. Identification of C18:1-phytoceramide as the candidate lipid mediator for hydroxyurea resistance in yeast. J Biol Chem. 2013; 288:17272-17284. [PubMed: 23620586]

51. Chauhan N, Visram M, Cristobal-Sarramian A, Sarkleti F, Kohlwein SD. Morphogenesis checkpoint kinase Swe1 is the executor of lipolysis-dependent cell-cycle progression. Proc Natl Acad Sci USA. 2015; 112:E1077-E1085. [PubMed: 25713391] 
52. Adada MM, et al. Intracellular sphingosine kinase 2-derived sphingosine-1-phosphate mediates epidermal growth factor-induced ezrin-radixin-moesin phosphorylation and cancer cell invasion. FASEB J. 2015; 29:4654-4669. [PubMed: 26209696]

53. Bretscher A, Edwards K, Fehon RG. ERM proteins and merlin: integrators at the cell cortex. Nat Rev Mol Cell Biol. 2002; 3:586-599. [PubMed: 12154370]

54. van der Weyden L, et al. Genome-wide in vivo screen identifies novel host regulators of metastatic colonization. Nature. 2017; 541:233-236. In an unbiased screen, this study identifies SPNS2, the S1P transporter, as a key regulator of metastasis. [PubMed: 28052056]

55. Romero-Guevara R, Cencetti F, Donati C, Bruni P. Sphingosine 1-phosphate signaling pathway in inner ear biology. New therapeutic strategies for hearing loss? Front Aging Neurosci. 2015; 7:60. [PubMed: 25954197]

56. Kitajiri S, et al. Radixin deficiency causes deafness associated with progressive degeneration of cochlear stereocilia. J Cell Biol. 2004; 166:559-570. [PubMed: 15314067]

57. Chen J, et al. Spinster homolog 2 (spns2) deficiency causes early onset progressive hearing loss. PLoS Genet. 2014; 10:e1004688. [PubMed: 25356849]

58. Canals D, Roddy P, Hannun YA. Protein phosphatase 1a mediates ceramide-induced ERM protein dephosphorylation: a novel mechanism independent of phosphatidylinositol 4, 5-biphosphate (PIP2) and myosin/ERM phosphatase. J Biol Chem. 2012; 287:10145-10155. [PubMed: 22311981]

59. Carreira AC, Ventura AE, Varela AR, Silva LC. Tackling the biophysical properties of sphingolipids to decipher their biological roles. Biol Chem. 2015; 396:597-609. [PubMed: 25581755]

60. Trajkovic K, et al. Ceramide triggers budding of exosome vesicles into multivesicular endosomes. Science. 2008; 319:1244-1247. This study ascribes a key role for ceramide and for nSMase 2 in the regulation of exocytosis. [PubMed: 18309083]

61. Kosaka N, et al. Secretory mechanisms and intercellular transfer of microRNAs in living cells. J Biol Chem. 2010; 285:17442-17452. [PubMed: 20353945]

62. Guo BB, Bellingham SA, Hill AF. The neutral sphingomyelinase pathway regulates packaging of the prion protein into exosomes. J Biol Chem. 2015; 290:3455-3467. [PubMed: 25505180]

63. Yuyama K, Sun H, Mitsutake S, Igarashi Y. Sphingolipid-modulated exosome secretion promotes clearance of amyloid-beta by microglia. J Biol Chem. 2012; 287:10977-10989. [PubMed: 22303002]

64. Shen H, et al. Coupling between endocytosis and sphingosine kinase 1 recruitment. Nat Cell Biol. 2014; 16:652-662. [PubMed: 24929359]

65. Hayashi Y, et al. Sphingomyelin synthase 2, but not sphingomyelin synthase 1, is involved in HIV-1 envelope-mediated membrane fusion. J Biol Chem. 2014; 289:30842-30856. [PubMed: 25231990]

66. Contreras FX, et al. Molecular recognition of a single sphingolipid species by a protein's transmembrane domain. Nature. 2012; 481:525-529. This study identifies a specific molecular species of sphingomyelin, C18 sphingomyelin, as a ligand for p24, a component of the COPI secretion machinery. [PubMed: 22230960]

67. Capasso S, et al. Sphingolipid metabolic flow controls phosphoinositide turnover at the trans-Golgi network. EMBO J. 2017; 36:1736-1754. [PubMed: 28495678]

68. Heffernan-Stroud LA, et al. Defining a role for sphingosine kinase 1 in p53-dependent tumors. Oncogene. 2012; 31:1166-1175. [PubMed: 21765468]

69. Wang Y, et al. Alkaline ceramidase 2 is a novel direct target of p53 and induces autophagy and apoptosis through ROS generation. Sci Rep. 2017; 7:44573. [PubMed: 28294157]

70. Shamseddine AA, et al. P53-dependent upregulation of neutral sphingomyelinase-2: role in doxorubicin-induced growth arrest. Cell Death Dis. 2015; 6:e1947. [PubMed: 26512957]

71. Guillas I, et al. C26-CoA-dependent ceramide synthesis of Saccharomyces cerevisiae is operated by Lag1p and Lac1p. EMBO J. 2001; 20:2655-2665. This study identifies the genes encoding CerSs (lagl and lacl) in yeast and demonstrates that these genes are in fact the first genes to be implicated in regulation of yeast lifespan. [PubMed: 11387200] 
72. Mosbech MB, et al. Functional loss of two ceramide synthases elicits autophagy-dependent lifespan extension in C. elegans. PLoS ONE. 2013; 8:e70087. [PubMed: 23894595]

73. Jazwinski SM, et al. HRAS1 and LASS1 with APOE are associated with human longevity and healthy aging. Aging Cell. 2010; 9:698-708. [PubMed: 20569235]

74. Dany M, Ogretmen B. Ceramide induced mitophagy and tumor suppression. Biochim Biophys Acta. 2015; 1853:2834-2845. [PubMed: 25634657]

75. Siddique MM, Li Y, Chaurasia B, Kaddai VA, Summers SA. Dihydroceramides: from bit players to lead actors. J Biol Chem. 2015; 290:15371-15379. This is an informative summary of the roles of ceramides and dihydroceramides in metabolic pathways. [PubMed: 25947377]

76. Hernandez-Tiedra S, et al. Dihydroceramide accumulation mediates cytotoxic autophagy of cancer cells via autolysosome destabilization. Autophagy. 2016; 12:2213-2229. [PubMed: 27635674]

77. Obeid LM, Linardic CM, Karolak LA, Hannun YA. Programmed cell death induced by ceramide. Science. 1993; 259:1769-1771. [PubMed: 8456305]

78. Siskind LJ, et al. The BCL-2 protein BAK is required for long-chain ceramide generation during apoptosis. J Biol Chem. 2010; 285:11818-11826. [PubMed: 20172858]

79. Brinkmann V, Lynch KR. FTY720: targeting G-protein-coupled receptors for sphingosine 1phosphate in transplantation and autoimmunity. Curr Opin Immunol. 2002; 14:569-575. [PubMed: 12183155]

80. Benechet AP, et al. T cell-intrinsic S1PR1 regulates endogenous effector T-cell egress dynamics from lymph nodes during infection. Proc Natl Acad Sci USA. 2016; 113:2182-2187. [PubMed: 26862175]

81. Hla T, Venkataraman K, Michaud J. The vascular S1P gradient-cellular sources and biological significance. Biochim Biophys Acta. 2008; 1781:477-482. [PubMed: 18674637]

82. Allende ML, Dreier JL, Mandala S, Proia RL. Expression of the sphingosine 1-phosphate receptor, S1P1, on T-cells controls thymic emigration. J Biol Chem. 2004; 279:15396-15401. [PubMed: 14732704]

83. Breart B, et al. Lipid phosphate phosphatase 3 enables efficient thymic egress. J Exp Med. 2011; 208:1267-1278. [PubMed: 21576386]

84. Blaho VA, et al. HDL-bound sphingosine-1-phosphate restrains lymphopoiesis and neuroinflammation. Nature. 2015; 523:342-346. This study demonstrates specific immune functions for HDL-bound S1P in the circulation. [PubMed: 26053123]

85. Pettus BJ, et al. The coordination of prostaglandin E2 production by sphingosine-1-phosphate and ceramide-1-phosphate. Mol Pharmacol. 2005; 68:330-335. [PubMed: 15900018]

86. Xiong Y, et al. Sphingosine kinases are not required for inflammatory responses in macrophages. J Biol Chem. 2016; 291:11465. [PubMed: 27226645]

87. Jenkins RW, et al. Regulation of CC ligand 5/RANTES by acid sphingomyelinase and acid ceramidase. J Biol Chem. 2011; 286:13292-13303. [PubMed: 21335555]

88. Kott M, et al. Acid sphingomyelinase serum activity predicts mortality in intensive care unit patients after systemic inflammation: a prospective cohort study. PLoS ONE. 2014; 9:e112323. [PubMed: 25384060]

89. Hannun, YA., Luberto, C., Mao, C., Obeid, LM. Bioactive Sphingolipids in Cancer Biology and Therapy. Springer; 2015.

90. Morad SA, Cabot MC. Ceramide-orchestrated signalling in cancer cells. Nat Rev Cancer. 2013; 13:51-65. [PubMed: 23235911]

91. Pettus BJ, et al. The sphingosine kinase 1/sphingosine-1-phosphate pathway mediates COX-2 induction and PGE2 production in response to TNF-a. FASEB J. 2003; 17:1411-1421. [PubMed: 12890694]

92. Tan SS, et al. Sphingosine kinase 1 promotes malignant progression in colon cancer and independently predicts survival of patients with colon cancer by competing risk approach in South asian population. Clin Transl Gastroenterol. 2014; 5:e51. [PubMed: 24572701]

93. Kawamori T, et al. Role for sphingosine kinase 1 in colon carcinogenesis. FASEB J. 2009; 23:405414. [PubMed: 18824518] 
94. Liang J, et al. Sphingosine-1-phosphate links persistent STAT3 activation, chronic intestinal inflammation, and development of colitis-associated cancer. Cancer Cell. 2013; 23:107-120. [PubMed: 23273921]

95. Kohno M, et al. Intracellular role for sphingosine kinase 1 in intestinal adenoma cell proliferation. Mol Cell Biol. 2006; 26:7211-7223. [PubMed: 16980623]

96. Oskouian B, et al. Sphingosine-1-phosphate lyase potentiates apoptosis via p53- and p38dependent pathways and is down-regulated in colon cancer. Proc Natl Acad Sci USA. 2006; 103:17384-17389. [PubMed: 17090686]

97. Ju T, Gao D, Fang ZY. Targeting colorectal cancer cells by a novel sphingosine kinase 1 inhibitor PF-543. Biochem Biophys Res Commun. 2016; 470:728-734. [PubMed: 26775841]

98. Chumanevich AA, et al. Suppression of colitis-driven colon cancer in mice by a novel small molecule inhibitor of sphingosine kinase. Carcinogenesis. 2010; 31:1787-1793. [PubMed: 20688834]

99. García-Barros M, et al. Role of neutral ceramidase in colon cancer. FASEB J. 2016; 30:4159-4171. [PubMed: 27609772]

100. Heffernan-Stroud LA, Obeid LM. Sphingosine kinase 1 in cancer. Adv Cancer Res. 2013; 117:201-235. [PubMed: 23290781]

101. Galvani S, et al. HDL-bound sphingosine 1-phosphate acts as a biased agonist for the endothelial cell receptor S1P1 to limit vascular inflammation. Sci Signal. 2015; 8:ra79. [PubMed: 26268607]

102. Nagahashi M, et al. Sphingosine-1-phosphate in chronic intestinal inflammation and cancer. Adv Biol Regul. 2014; 54:112-120. [PubMed: 24210073]

103. Anelli V, Gault CR, Snider AJ, Obeid LM. Role of sphingosine kinase-1 in paracrine/transcellular angiogenesis and lymphangiogenesis in vitro. FASEB J. 2010; 24:2727-2738. [PubMed: 20335228]

104. Mahdy AE, et al. Acid ceramidase upregulation in prostate cancer cells confers resistance to radiation: AC inhibition, a potential radiosensitizer. Mol Ther. 2009; 17:430-438. [PubMed: 19107118]

105. Frohbergh M, He X, Schuchman EH. The molecular medicine of acid ceramidase. Biol Chem. 2015; 396:759-765. [PubMed: 25938220]

106. Realini N, et al. Acid ceramidase in melanoma: expression, localization, and effects of pharmacological inhibition. J Biol Chem. 2016; 291:2422-2434. [PubMed: 26553872]

107. Bizzozero L, et al. Acid sphingomyelinase determines melanoma progression and metastatic behaviour via the microphtalmia-associated transcription factor signalling pathway. Cell Death Differ. 2014; 21:507-520. [PubMed: 24317198]

108. Sanger N, et al. Acid ceramidase is associated with an improved prognosis in both DCIS and invasive breast cancer. Mol Oncol. 2015; 9:58-67. [PubMed: 25131496]

109. Carpinteiro A, et al. Regulation of hematogenous tumor metastasis by acid sphingomyelinase. EMBO Mol Med. 2015; 7:714-734. [PubMed: 25851537]

110. Truman JP, et al. Endothelial membrane remodeling is obligate for anti-angiogenic radiosensitization during tumor radiosurgery. PLoS ONE. 2010; 5:e12310. [PubMed: 20808818]

111. Daemen A, et al. Metabolite profiling stratifies pancreatic ductal adenocarcinomas into subtypes with distinct sensitivities to metabolic inhibitors. Proc Natl Acad Sci USA. 2015; 112:E4410E4417. [PubMed: 26216984]

112. Dubois N, et al. Plasma ceramide, a real-time predictive marker of pulmonary and hepatic metastases response to stereotactic body radiation therapy combined with irinotecan. Radiother Oncol. 2016; 119:229-235. [PubMed: 27113798]

113. Abdul Aziz NA, et al. 19-Gene expression signature as a predictor of survival in colorectal cancer. BMC Med Genom. 2016; 9:58. This study identifies CERS6 as a key gene component of a 19gene signature for prediction of survival in colon cancer.

114. Kasumov T, et al. Ceramide as a mediator of non-alcoholic fatty liver disease and associated atherosclerosis. PLoS ONE. 2015; 10:e0126910. [PubMed: 25993337]

115. Boini KM, Zhang C, Xia M, Poklis JL, Li PL. Role of sphingolipid mediator ceramide in obesity and renal injury in mice fed a high-fat diet. J Pharmacol Exp Ther. 2010; 334:839-846. [PubMed: 20543095] 
116. Choi S, Snider AJ. Sphingolipids in high fat diet and obesity-related diseases. Mediators Inflamm. 2015; 2015:520618. [PubMed: 26648664]

117. Hodson AE, Tippetts TS, Bikman BT. Insulin treatment increases myocardial ceramide accumulation and disrupts cardiometabolic function. Cardiovasc Diabetol. 2015; 14:153. [PubMed: 26682540]

118. Kurek K, et al. Inhibition of ceramide de novo synthesis with myriocin affects lipid metabolism in the liver of rats with streptozotocin-induced type 1 diabetes. BioMed Res Int. 2014; 2014:980815. [PubMed: 24701589]

119. Turpin SM, et al. Obesity-induced CerS6-dependent C16:0 ceramide production promotes weight gain and glucose intolerance. Cell Metab. 2014; 20:678-686. [PubMed: 25295788]

120. Xia JY, et al. Targeted induction of ceramide degradation leads to improved systemic metabolism and reduced hepatic steatosis. Cell Metab. 2015; 22:266-278. [PubMed: 26190650]

121. Chavez JA, et al. Ceramides and glucosylceramides are independent antagonists of insulin signaling. J Biol Chem. 2014; 289:723-734. [PubMed: 24214972]

122. Li Z, et al. Reducing plasma membrane sphingomyelin increases insulin sensitivity. Mol Cell Biol. 2011; 31:4205-4218. [PubMed: 21844222]

123. Yano M, et al. Increased oxidative stress impairs adipose tissue function in sphingomyelin synthase 1 null mice. PLoS ONE. 2013; 8:e61380. [PubMed: 23593476]

124. Taguchi Y, et al. Sphingosine-1-phosphate phosphatase 2 regulates pancreatic islet $\beta$-cell endoplasmic reticulum stress and proliferation. J Biol Chem. 2016; 291:12029-12038. [PubMed: 27059959]

125. Chen J, et al. Deletion of sphingosine kinase 1 ameliorates hepatic steatosis in diet-induced obese mice: role of PPAR $\gamma$. Biochim Biophys Acta. 2016; 1861:138-147. [PubMed: 26615875]

126. Park K, et al. ER stress stimulates production of the key antimicrobial peptide, cathelicidin, by forming a previously unidentified intracellular S1P signaling complex. Proc Natl Acad Sci USA. 2016; 113:E1334-E1342. [PubMed: 26903652]

127. Wong ML, et al. Acute systemic inflammation up-regulates secretory sphingomyelinase in vivo: A possible link between inflammatory cytokines and atherogenesis. Proc Natl Acad Sci USA. 2000; 97:8681-8686. [PubMed: 10890909]

128. Fan J, Wu BX, Crosson CE. Suppression of acid sphingomyelinase protects the retina from ischemic injury. Invest Ophthalmol Vis Sci. 2016; 57:4476-4484. [PubMed: 27571014]

129. Reforgiato MR, et al. Inhibition of ceramide de novo synthesis as a postischemic strategy to reduce myocardial reperfusion injury. Basic Res Cardiol. 2016; 111:12. [PubMed: 26786259]

130. Hammad SM, et al. Increased plasma levels of select deoxy-ceramide and ceramide species are associated with increased odds of diabetic neuropathy in type 1 diabetes: a pilot study. Neuromolecular Med. 2017; 19:46-56. [PubMed: 27388466]

131. Havulinna AS, et al. Circulating ceramides predict cardiovascular outcomes in the populationbased FINRISK 2002 cohort. Arterioscler Thromb Vasc Biol. 2016; 36:2424-2430. [PubMed: 27765765]

132. Cheng JM, et al. Plasma concentrations of molecular lipid species in relation to coronary plaque characteristics and cardiovascular outcome: results of the ATHEROREMO-IVUS study. Atherosclerosis. 2015; 243:560-566. [PubMed: 26523994]

133. Sigruener A, et al. Glycerophospholipid and sphingolipid species and mortality: the Ludwigshafen Risk and Cardiovascular Health (LURIC) study. PLoS ONE. 2014; 9:e85724. [PubMed: 24465667]

134. Saleem M, et al. Ceramides predict verbal memory performance in coronary artery disease patients undertaking exercise: a prospective cohort pilot study. BMC Geriatr. 2013; 13:135. [PubMed: 24330446]

135. Soltau I, et al. Serum-sphingosine-1-phosphate concentrations are inversely associated with atherosclerotic diseases in humans. PLoS ONE. 2016; 11:e0168302. [PubMed: 27973607]

136. Othman A, et al. Plasma 1-deoxysphingolipids are predictive biomarkers for type 2 diabetes mellitus. BMJ Open Diabetes Res Care. 2015; 3:e000073.

137. Hama H. Fatty acid 2-hydroxylation in mammalian sphingolipid biology. Biochim Biophys Acta. 2010; 1801:405-414. [PubMed: 20026285] 
138. Edvardson S, et al. Deficiency of the alkaline ceramidase ACER3 manifests in early childhood by progressive leukodystrophy. J Med Genet. 2016; 53:389-396. [PubMed: 26792856]

139. Zhao L, et al. Elevation of 20-carbon long chain bases due to a mutation in serine palmitoyltransferase small subunit b results in neurodegeneration. Proc Natl Acad Sci USA. 2015; 112:12962-12967. [PubMed: 26438849]

140. Vanni N, et al. Impairment of ceramide synthesis causes a novel progressive myoclonus epilepsy. Ann Neurol. 2014; 76:206-212. [PubMed: 24782409]

141. Mosbech MB, et al. Reduced ceramide synthase 2 activity causes progressive myoclonic epilepsy. Ann Clin Transl Neurol. 2014; 1:88-98. [PubMed: 25356388]

142. Boustany RM. Ceramide center stage in progressive myoclonus epilepsies. Ann Neurol. 2014; 76:162-164. [PubMed: 25041902]

143. Spassieva SD, et al. Ectopic expression of ceramide synthase 2 in neurons suppresses neurodegeneration induced by ceramide synthase 1 deficiency. Proc Natl Acad Sci USA. 2016; 113:5928-5933. This study, by using genetic interactions between Cers 1 and Cers2, demonstrates that sphingosine is likely the key lipid species responsible for mediating neurodegeneration in the Cers1-knockout mouse. [PubMed: 27162368]

144. Dinkins MB, et al. Neutral sphingomyelinase-2 deficiency ameliorates Alzheimer's disease pathology and improves cognition in the 5XFAD mouse. J Neurosci. 2016; 36:8653-8667. [PubMed: 27535912]

145. Novgorodov SA, et al. Essential roles of neutral ceramidase and sphingosine in mitochondrial dysfunction due to traumatic brain injury. J Biol Chem. 2014; 289:13142-13154. [PubMed: 24659784]

146. Jennemann R, et al. Loss of ceramide synthase 3 causes lethal skin barrier disruption. Hum Mol Genet. 2012; 21:586-608. [PubMed: 22038835]

147. Behne M, et al. Omega-hydroxyceramides are required for corneocyte lipid envelope (CLE) formation and normal epidermal permeability barrier function. J Invest Dermatol. 2000; 114:185-192. [PubMed: 10620136]

148. Jennemann R, et al. Integrity and barrier function of the epidermis critically depend on glucosylceramide synthesis. J Biol Chem. 2007; 282:3083-3094. [PubMed: 17145749]

149. Westerberg R, et al. Role for ELOVL3 and fatty acid chain length in development of hair and skin function. J Biol Chem. 2004; 279:5621-5629. [PubMed: 14581464]

150. Cameron DJ, et al. Essential role of Elovl4 in very long chain fatty acid synthesis, skin permeability barrier function, and neonatal survival. Int J Biol Sci. 2007; 3:111-119. [PubMed: 17304340]

151. Peters F, et al. Ceramide synthase 4 regulates stem cell homeostasis and hair follicle cycling. J Invest Dermatol. 2015; 135:1501-1509. [PubMed: 25705848]

152. Liakath-Ali K, et al. Alkaline ceramidase 1 is essential for mammalian skin homeostasis and regulating whole-body energy expenditure. J Pathol. 2016; 239:374-383. [PubMed: 27126290]

153. Stoffel W, Jenke B, Block B, Zumbansen M, Koebke J. Neutral sphingomyelinase 2 (smpd3) in the control of postnatal growth and development. Proc Natl Acad Sci USA. 2005; 102:45544559. [PubMed: 15764706]

154. Li J, et al. Smpd3 expression in both chondrocytes and osteoblasts is required for normal endochondral bone development. Mol Cell Biol. 2016; 36:2282-2299. [PubMed: 27325675]

155. Kakoi H, et al. Bone morphogenic protein (BMP) signaling up-regulates neutral sphingomyelinase 2 to suppress chondrocyte maturation via the Akt protein signaling pathway as a negative feedback mechanism. J Biol Chem. 2014; 289:8135-8150. [PubMed: 24505141]

156. Somenzi G, et al. Disruption of retinoic acid receptor alpha reveals the growth promoter face of retinoic acid. PLoS ONE. 2007; 2:e836. [PubMed: 17786207]

157. Clarke CJ, et al. ATRA transcriptionally induces nSMase 2 through $\mathrm{CBP} / \mathrm{p} 300$-mediated histone acetylation. J Lipid Res. 2016; 57:868-881. [PubMed: 27013100]

158. Cowart LA, Hannun YA. Selective substrate supply in the regulation of yeast de novo sphingolipid synthesis. J Biol Chem. 2007; 282:12330-12340. [PubMed: 17322298] 
159. Sun Y, et al. Orm protein phosphoregulation mediates transient sphingolipid biosynthesis response to heat stress via the Pkh-Ypk and Cdc55-PP2A pathways. Mol Biol Cell. 2012; 23:2388-2398. [PubMed: 22535525]

160. Muir A, Ramachandran S, Roelants FM, Timmons G, Thorner J. TORC2-dependent protein kinase Ypk1 phosphorylates ceramide synthase to stimulate synthesis of complex sphingolipids. eLife. 2014; 3:e03779.

161. Novgorodov SA, et al. SIRT3 deacetylates ceramide synthases: implications for mitochondrial dysfunction and brain injury. J Biol Chem. 2016; 291:1957-1973. [PubMed: 26620563]

162. Sassa T, Hirayama T, Kihara A. Enzyme activities of the ceramide synthases CERS2-6 are regulated by phosphorylation in the C-terminal region. J Biol Chem. 2016; 291:7477-7487. [PubMed: 26887952]

163. Jensen SA, et al. Bcl2L13 is a ceramide synthase inhibitor in glioblastoma. Proc Natl Acad Sci USA. 2014; 111:5682-5687. [PubMed: 24706805]

164. McNaughton M, Pitman M, Pitson SM, Pyne NJ, Pyne S. Proteasomal degradation of sphingosine kinase 1 and inhibition of dihydroceramide desaturase by the sphingosine kinase inhibitors, SKi or ABC294640, induces growth arrest in androgen-independent LNCaP-AI prostate cancer cells. Oncotarget. 2016; 7:16663-16675. [PubMed: 26934645]

165. Filosto S, Ashfaq M, Chung S, Fry W, Goldkorn T. Neutral sphingomyelinase 2 activity and protein stability are modulated by phosphorylation of five conserved serines. J Biol Chem. 2012; 287:514-522. [PubMed: 22074919]

166. Shamseddine AA, Airola MV, Hannun YA. Roles and regulation of neutral sphingomyelinase-2 in cellular and pathological processes. Adv Biol Reg. 2015; 57:24-41.

167. Rhein C, et al. Functional implications of novel human acid sphingomyelinase splice variants. PLoS ONE. 2012; 7:e35467. [PubMed: 22558155]

168. Sasaki H, et al. Regulation of alkaline ceramidase activity by the c-Src-mediated pathway. Arch Biochem Biophys. 2014; 550-551:12-19.

169. Tanaka K, et al. Role of down-regulated neutral ceramidase during all-trans retinoic acid-induced neuronal differentiation in SH-SY5Y neuroblastoma cells. J Biochem. 2012; 151:611-620. [PubMed: 22451680]

170. Wu BX, Zeidan YH, Hannun YA. Downregulation of neutral ceramidase by gemcitabine: Implications for cell cycle regulation. Biochim Biophys Acta. 2009; 1791:730-739. [PubMed: 19345744]

171. Rahmaniyan M, et al. Identification of dihydroceramide desaturase as a direct in vitro target for fenretinide. J Biol Chem. 2011; 286:24754-24764. This study identifies dihydroceramide desaturase, the enzyme responsible for introducing the 4-5 double bond into ceramide, as a direct target for the action of the chemotherapeutic agent fenretinide (4-HPR). [PubMed: 21543327]

172. Schnute ME, et al. Modulation of cellular S1P levels with a novel, potent and specific inhibitor of sphingosine kinase-1. Biochem J. 2012; 444:79-88. [PubMed: 22397330]

173. Rex K, et al. Sphingosine kinase activity is not required for tumor cell viability. PLoS ONE. 2013; 8:e68328. [PubMed: 23861887]

174. Santos WL, Lynch KR. Drugging sphingosine kinases. ACS Chem Biol. 2015; 10:225-233. [PubMed: 25384187]

175. Realini N, et al. Discovery of highly potent acid ceramidase inhibitors with in vitro tumor chemosensitizing activity. Sci Rep. 2013; 3:1035. [PubMed: 23301156]

176. Sandborn WJ, et al. Ozanimod induction and maintenance treatment for ulcerative colitis. N Engl J Med. 2016; 374:1754-1762. [PubMed: 27144850]

177. Zhang L, et al. Anti-S1P antibody as a novel therapeutic strategy for VEGFR TKI-resistant renal cancer. Clin Cancer Res. 2015; 21:1925-1934. [PubMed: 25589614]

178. Rollin-Pinheiro R, Singh A, Barreto-Bergter E, Del Poeta M. Sphingolipids as targets for treatment of fungal infections. Future Med Chem. 2016; 8:1469-1484. [PubMed: 27502288]

179. Kumagai K, Kawano-Kawada M, Hanada K. Phosphoregulation of the ceramide transport protein CERT at serine 315 in the interaction with VAMP-associated protein (VAP) for inter-organelle trafficking of ceramide in mammalian cells. J Biol Chem. 2014; 289:10748-10760. [PubMed: 24569996] 
180. D'Angelo G, et al. Glycosphingolipid synthesis requires FAPP2 transfer of glucosylceramide. Nature. 2007; 449:62-67. This study identifies that the transfer protein FAPP2 is involved in the selective binding and transport of neutral glycolipids among Golgi cisternae. [PubMed: 17687330] 
A
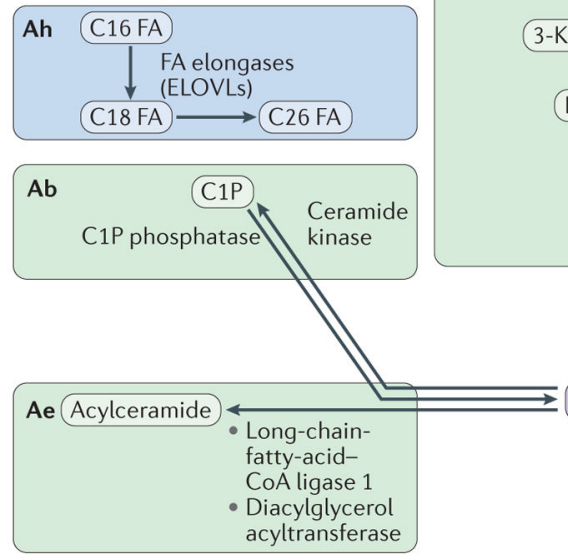

Ac Sphingomyelin Sphingomyelinase synthase $\downarrow$

Sphingomyelin

Dihydroceramide
Aa Serine + Palmitoyl-CoA (Alanine or glycine + stearate or myristate) Serine

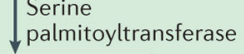

3-Ketodihydrosphingosine $\downarrow 3$-Ketoreductase

Dihydrosphingosine

Dihydroceramide

Desaturase

\section{se} se

,

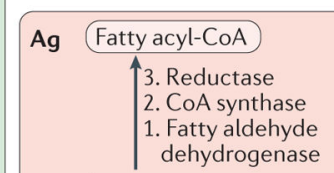

Fatty aldehydes + Ethanolamine phosphate

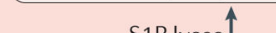
(10)

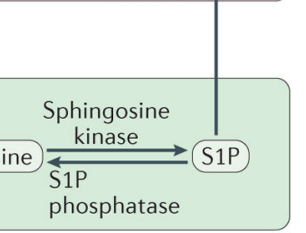

synthase

phosphatase

Glucosylceramide Acid $\beta$-glucosylceramidase synthase

Ad $\frac{\downarrow}{\text { Glycosphingolipids }}$

B

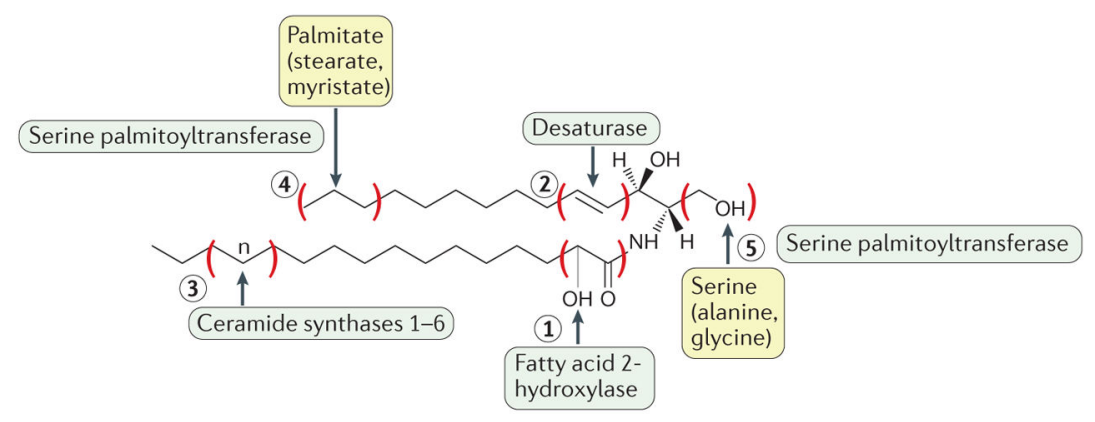

Figure 1. Overview of sphingolipid metabolism

It is convenient to envision sphingolipid metabolism as organized into blocks. Aa $\mid$ The de novo biosynthetic pathway is initiated in the endoplasmic reticulum by the action of serine palmitoyltransferase (SPT). Sequential reactions lead to the generation of ceramides. Preferential substrates include serine and palmitoyl-CoA, but alanine or glycine and stearate or myristate can also be used. Ab-Ad | Ceramides are then incorporated into various complex sphingolipids (predominantly in the Golgi) through modifications at the 1-hydroxyl position to generate ceramide-1-phosphate (C1P), sphingomyelin or glycoceramides, which in turn serve as the precursors for the various glycosphingolipids. Ae | Ceramides can be acylated to form 1- $O$-acyl-ceramides. Af $\mid$ In sphingolipid catabolic pathways, sphingomyelin, C1P and glycosphingolipids are hydrolysed, resulting in the formation of ceramide (see parts $\mathbf{b}, \mathbf{c}$ and $\mathbf{d}$ ). Constitutive catabolism occurs in the lysosome. Ceramide can then be deacylated to generate sphingosine, which in turn is phosphorylated to sphingosine-1-phosphate (S1P). Ag | Exit from sphingolipids is initiated by the action of S1P lyase, which cleaves S1P (or dihydroS1P) to a fatty aldehyde and ethanolamine phosphate. The fatty aldehyde undergoes further metabolism, resulting in the formation of palmitoyl-CoA. Ah | The fatty acid (FA) elongation module is an important adjunct 
component of the sphingolipid pathway, as sphingolipids are the primary lipids with verylong (C22-26) and ultra-long (longer than C26) fatty acyl chains. B | As the precursor of all complex sphingolipids, ceramides constitute a family of closely related molecules that contain a sphingoid base and an amino-linked FA. Different enzymes introduce variations to the basic structure. Fatty acid 2-hydroxylase introduces an $\mathrm{OH}$ on the fatty acyl group (variation 1). Sphingolipids (4)-desaturase DES1 (DEGS1) introduces a double bond in the 4-5 position of the sphingoid base (variation 2), whereas DEGS2 can introduce an $\mathrm{OH}$ on position 4 (not shown). Ceramide synthases introduce fatty acyl groups of distinct chain length (variation 3). SPT can utilize myristate or stearate instead of palmitate, thus introducing variations in the length of the sphingoid backbone (variation 4). SPT can also utilize alanine or glycine, thus introducing variation 5. For easy nomenclature of ceramides, the chain length of the fatty acyl group is indicated (for example, C18 Cer for the $N$-stearoyl ceramide). More complete nomenclature indicates the sphingoid base length and degree of FA chain desaturation, for example, 18:1C18 ceramide refers to ceramide with canonical sphingosine and stearate in amide linkage. Non-chemical terminology can also be used, for example, dihydroCer refers to ceramides lacking the 4-5 double bond. ELOVLs, elongation of very-long-chain fatty acids proteins. 


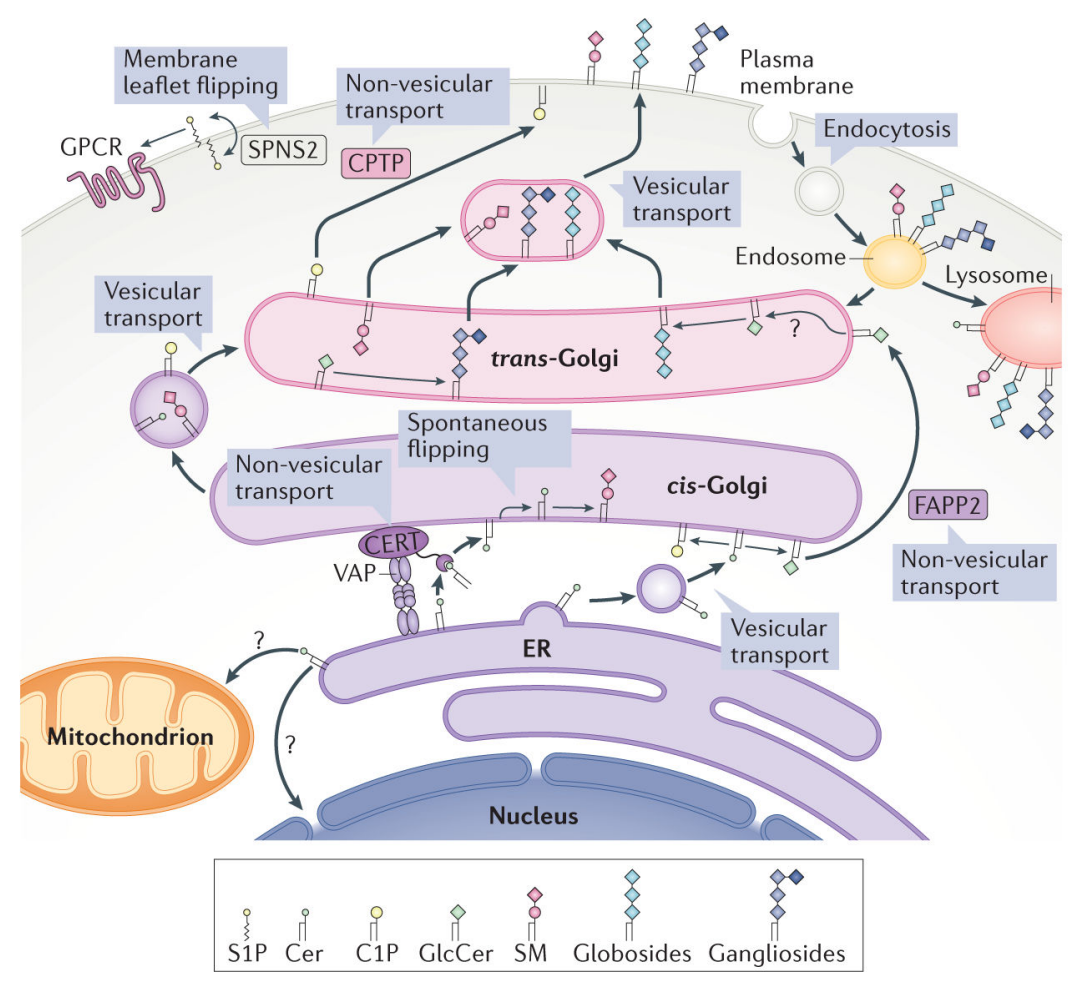

Figure 2. Intracellular compartmentalization and transport of sphingolipids

It is now becoming clear that the cellular actions of bioactive sphingolipids are 'local'. Therefore, the site of production of these lipids is critical, and it is determined by the location of the specific enzymes involved in the regulatory pathways. For details of the compartmentalization of these processes, please refer to box 1 in REF. 1. Moreover, the metabolic and cellular functions of bioactive lipids are further controlled by specific translocases and lipid transport proteins. A strong case can now be made that ceramides (Cers) generated in the endoplasmic reticulum (ER) can reach the Golgi either by a vesicular route, where they are coupled to the synthesis of glucosylceramide, or by specific translocation via the action of ceramide transfer protein (CERT), which couples ceramides to the synthesis of sphingomyelin $(\mathrm{SM})^{179}$. Likewise, the transfer protein phosphatidylinositolfour-phosphate adaptor protein 2 (FAPP2) can transfer glucosylceramide (GlcCer) between Golgi networks and couple it specifically to the synthesis of globosides (globo series of neutral glycosphingolipids) as opposed to the anionic ganglio series ${ }^{180}$. Ceramide- 1 phosphate $(\mathrm{C} 1 \mathrm{P})$ is the target of the non-vesicular translocation activity of C1P transfer protein (CPTP), which is involved in transferring it from the Golgi to other compartments such as the plasma membrane ${ }^{35}$. Protein spinster homologue 2 (SPNS2) flips sphingosine-1phosphate (S1P) across the plasma membrane to deliver it to exocytoplasmic leaflets, where it can access its receptors (a subfamily of $G$ protein-coupled receptors (GPCRs) ${ }^{22}$. VAP, vesicle-associated membrane protein-associated protein. 

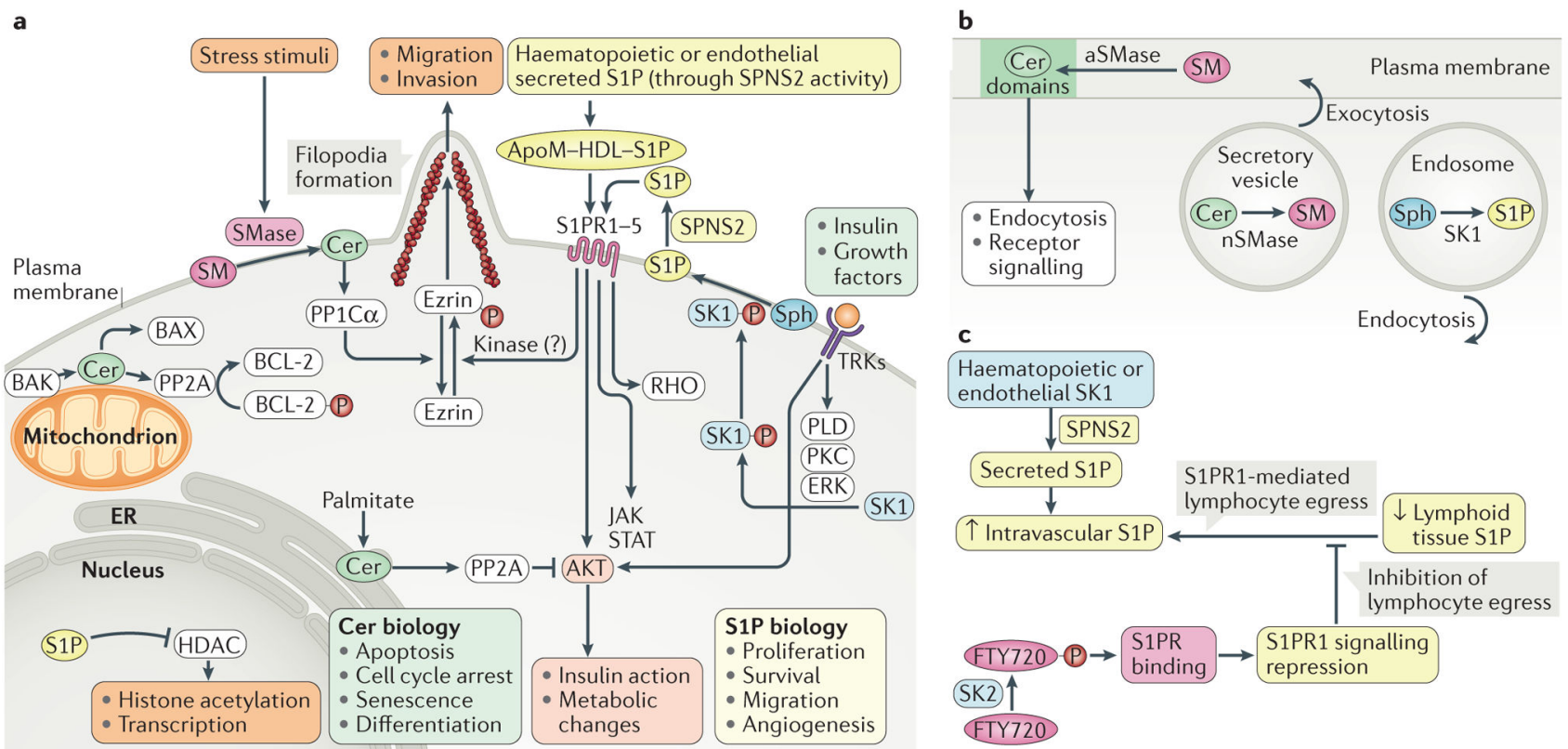

Figure 3. Examples of cellular functions and downstream targets of bioactive sphingolipids a Several factors, including insulin and growth factors, have been shown to activate sphingosine kinases (SKs) in a mechanism involving their phosphorylation in a phospholipase D (PLD)-dependent, protein kinase C (PKC)-dependent and ERK-dependent manner, resulting in the formation of sphingosine-1-phosphate (S1P) from sphingosine (Sph) intracellularly. S1P has to flip extra-cytosolically (with the involvement of protein spinster homologue 2 (SPNS2)) in order to interact with one (or more) of its five receptors (S1P receptor 1-5 (S1PR1-5)). S1P can also be released from the plasma membrane into the blood, and it is present in relatively high levels in the circulation. S1PRs transmit signals to key downstream targets such as serine/threonine protein kinase AKT, RHO and the RasERK and tyrosine protein kinase JAK-signal transducer and activator of transcription (STAT) pathways. Signalling through S1PRs also has other cellular targets. For example, S1PR actions result in robust phosphorylation of ezrin, which affects actin dynamics, promoting filopodia formation and cell migration (including cancer cell invasion). S1P also has nuclear actions - it binds and inhibits histone deacetylases (HDACs), leading to alterations in protein (histone) acetylation ${ }^{38}$. Ceramides (Cers) can be formed in several compartments. At the plasma membrane, ceramide generation from the action of sphingomyelinases (SMases) results in activation of protein phosphatase PP1Ca, which induces dephosphorylation of its substrates, including ezrin. Ceramide formed metabolically from the supply of palmitate can activate PP2A phosphatases to result in dephosphorylation and inactivation of AKT. Activation of PP2A by ceramide also occurs in the context of apoptosis. In this case, the pro-apoptotic protein Bcl-2 homologous antagonist/killer (BAK) promotes ceramide synthesis (through activation of ceramide synthase (CerS)), followed by PP2A activation and dephosphorylation and inactivation of anti-apoptotic BCL-2 proteins. In addition, mitochondrial ceramide was shown to promote the activity of the apoptosis regulator BAX, a pro-apoptotic protein. b | Bioactive lipids are increasingly appreciated to regulate membrane dynamics. Formation of ceramide at the plasma membrane by acid 
SMase (aSMase) has been shown to induce formation of distinct membrane domains that affect endocytosis and receptor signalling (for example, tumour necrosis factor receptor superfamily member 6 (FAS)) signalling. Neutral SMase2 (nSMase) and ceramide have been implicated in exocytosis, and SK1 and S1P have been implicated in endocytosis. c Egress of lymphocytes from lymphoid tissues to the blood is controlled by a gradient of S1P (high in blood and low in lymphoid tissue). The response to this gradient is primarily driven by S1PRs, especially S1PR1. The clinical compound FTY720 is converted to the active FTY720 phosphate through phosphorylation mediated by SK2 to suppress S1PR1 function and therefore lymphoid egress. ApoM, apolipoprotein M; ER, endoplasmic reticulum; HDL, high-density lipoprotein; TRK, tyrosine kinase receptor. 


\section{Table 1}

Sphingolipid species and their key cellular functions

\begin{tabular}{|c|c|}
\hline Cellular process & Sphingolipid species (and enzymes) involved \\
\hline \multicolumn{2}{|l|}{ Ceramide } \\
\hline Cell death & $\begin{array}{l}\text { - } \uparrow \mathrm{C} 18 \text { ceramide in chemotherapy-induced cell death in human HNSCCs } \\
\text { - } \uparrow \mathrm{C} 16 \text { ceramide via CerS activation induced by a variety of cell stressors } \\
\text { - Exogenous C16 ceramide } \\
\text { - } \uparrow \mathrm{C} 16 \text { and C24 ceramide in neutrophils } \\
\text { - } \downarrow \mathrm{C} 16 \text { ceramide via CerS6 downregulation in human HNSCCs } \\
\text { - } \uparrow \mathrm{C} 16 \text { ceramide induced by ACDase inhibition } \\
\text { - aSMase-dependent C16 ceramide generation }\end{array}$ \\
\hline Cell differentiation & - $\uparrow$ Total ceramides \\
\hline $\begin{array}{l}\text { Stimulation of cell migration and/or } \\
\text { invasion in cancer cells }\end{array}$ & $\begin{array}{l}\text { - } \downarrow \text { C16 ceramide via downregulation of CERS6 during epithelial-to-mesenchymal transition } \\
\text { - } \uparrow \text { Ceramides in arsenic trioxide in HCCLM3 hepatocarcinoma cells }\end{array}$ \\
\hline Cell proliferation & - $\uparrow \mathrm{C} 24$ and $\mathrm{C} 24: 1$ ceramide via overexpression of CerS2 \\
\hline Cell cycle arrest & - Exogenous $\mathrm{C} 2$ or C6 ceramide \\
\hline Senescence & $\begin{array}{l}\text { - } \uparrow \text { Total ceramides } \\
\text { - Exogenous C8 ceramide }\end{array}$ \\
\hline Necrosis & $\begin{array}{l}\text { - } \uparrow \text { Total ceramides } \\
\text { - Exogenous C2 or C6 ceramide }\end{array}$ \\
\hline Necroptosis & $\begin{array}{l}\text { - Exogenous C16 ceramide } \\
\text { - } \uparrow \mathrm{C} 16 \text { ceramide in } \mathrm{TNFa} \text {-treated cells }\end{array}$ \\
\hline Autophagy & $\begin{array}{l}\text { - } \uparrow \text { Ceramide due to cell stressor } \\
\text { - Exogenous C2 or C6 ceramide in tamoxifen-treated cells } \\
\text { - } \uparrow \mathrm{C} 16 \text { ceramide }\end{array}$ \\
\hline Mitophagy & - $\uparrow \mathrm{C} 18$ ceramide via CerS1 activity \\
\hline Cytoskeleton rearrangement & - $\uparrow$ Total ceramides via activation of aSMase or exogenous bSMase \\
\hline $\begin{array}{l}\text { Insulin resistance and cellular } \\
\text { metabolism }\end{array}$ & $\begin{array}{l}\text { - } \uparrow \text { Total ceramides in high-fat diet administration and/or palmitate treatment via CerS, nSMase or } \\
\text { aSMase activity } \\
\text { - } \uparrow \mathrm{C} 16 \text { ceramide due to CerS6 upregulation upon high-fat diet administration } \\
\text { - Exogenous C2 or C6 ceramides }\end{array}$ \\
\hline \multicolumn{2}{|l|}{$S 1 P$} \\
\hline Cell survival & • $\uparrow \mathrm{S} 1 \mathrm{P}$ \\
\hline Autophagy & $\begin{array}{l}\text { - Exogenous S1P } \\
\cdot \uparrow \mathrm{S} 1 \mathrm{P} \text { via overexpression of SK1 during cell starvation }\end{array}$ \\
\hline Inflammation & $\begin{array}{l}\text { - } \uparrow S 1 P \text { in TNFa-treated cells } \\
\text { - } \uparrow \text { S1P in S1P lyase-deficient mice } \\
\text { - } \uparrow \text { S1P via upregulation of SK1 }\end{array}$ \\
\hline Cell migration and invasion & $\begin{array}{l}\text { - } \uparrow S 1 P \text { via SK2 activation during EGF stimulation } \\
\text { - } \uparrow \text { S1P via overexpression of SK2 or ACER2 } \\
\text { - } \uparrow \text { S1P via SK1 mRNA and/or protein expression in cancer cells } \\
\text { - Exogenous S1P }\end{array}$ \\
\hline Cytoskeleton rearrangement & $\begin{array}{l}\text { - } \uparrow S 1 P \text { in HeLa cells treated with bSMase or NCDase } \\
\text { - } \uparrow \text { S1P in EGF-treated cells } \\
\text { - Exogenous S1P } \\
\text { - } \uparrow \text { Total ceramide via activation of aSMase in cisplatin-treated cells }\end{array}$ \\
\hline \multicolumn{2}{|l|}{ Sphingosine } \\
\hline Apoptosis & $\begin{array}{l}\text { - } \uparrow \text { Sphingosine in cancer cells induced by environmental stress, chemotherapeutic treatment and } \\
\text { apoptotic stimulus } \\
\text { - Exogenous sphingosine treatment } \\
\text { - } \uparrow \text { Sphingosine via SK inhibition }\end{array}$ \\
\hline Cell cycle arrest & $\begin{array}{l}\text { - } \uparrow \text { Sphingosine during DNA damage } \\
\text { - } \uparrow \text { Sphingosine via upregulation of ACER2 }\end{array}$ \\
\hline
\end{tabular}




\begin{tabular}{|c|c|}
\hline Cellular process & Sphingolipid species (and enzymes) involved \\
\hline & • Exogenous sphingosine \\
\hline Cell differentiation & $\begin{array}{l}\text { - } \uparrow \text { Sphingosine via upregulation of ACER } 1 \text { and ACDase in human epidermal keratinocytes } \\
\text { - Exogenous sphingosine }\end{array}$ \\
\hline \multicolumn{2}{|l|}{ C1P } \\
\hline Cell migration & $\begin{array}{l}-\uparrow C 1 P \text { by upregulation of CERK } \\
\text { - Exogenous C1P treatment }\end{array}$ \\
\hline Cell proliferation & $\begin{array}{l}- \text { Exogenous C1P } \\
\cdot \uparrow \mathrm{C} 1 \mathrm{P} \text { in cells overexpressing CERK } \\
\cdot \uparrow \mathrm{C} 1 \mathrm{P} \text { production in cells cultured in medium supplemented with fetal bovine serum }\end{array}$ \\
\hline Inhibition of apoptosis & $\cdot$ Exogenous $\mathrm{C} 1 \mathrm{P}$ \\
\hline Regulation of inflammation & $\begin{array}{l}\text { - Exogenous C1P } \\
\text { - } \uparrow 1 \mathrm{P} \text { via CERK upregulation upon IL-1 } \beta \text { or TNFa treatment or stimulation of resting macrophages } \\
\text { with macrophage colony-stimulating factor }\end{array}$ \\
\hline \multicolumn{2}{|l|}{ Dihydroceramide } \\
\hline Cell cycle arrest & - $\uparrow$ Total dihydroceramides via downregulation of $D E G S 1$ \\
\hline Apoptosis & $\begin{array}{l}-\uparrow \mathrm{C} 16 \text { dihydroceramide } \\
\cdot \uparrow \mathrm{C} 16 \text { dihydroceramide in 4-HPR-treated cells } \\
\cdot \uparrow \mathrm{C} 22 \text { and } \mathrm{C} 24 \text { dihydroceramide in T cell acute lymphoblastic leukaemia cell lines }\end{array}$ \\
\hline Inhibition of cell growth & $\begin{array}{l}\text { - } \uparrow \mathrm{C} 16 \text { dihydroceramide induced by the SK2 inhibitor ABC294640 in TRAMP-C2 cells } \\
\text { - } \uparrow \text { Total dihydroceramides via downregulation of DEGS1 or DEGS2 or direct treatment with } \\
\text { dihydroceramides }\end{array}$ \\
\hline Autophagy & $\begin{array}{l}\text { - } \uparrow \text { Total dihydroceramides } \\
\text { - } \uparrow \text { C16 dihydroceramide in resveratrol-treated HGC-27 human gastric cancer cells }\end{array}$ \\
\hline \multicolumn{2}{|l|}{ Sphingomyelin } \\
\hline Cell growth & $\begin{array}{l}\text { - } \uparrow \text { Sphingomyelin via SMS1 overexpression } \\
\text { • Exogenous sphingomyelin } \\
\text { - } \uparrow \text { Sphingomyelin via basic fibroblast growth factor-dependent stimulation of SMS }\end{array}$ \\
\hline Cell adhesion & $\begin{array}{l}\text { - } \uparrow \text { Sphingomyelin in cells treated with phorbol ester stimulated cell adhesion } \\
\text { - } \downarrow \text { Sphingomyelin induced by nSMase treatment caused detachment }\end{array}$ \\
\hline \multicolumn{2}{|l|}{ Galactosylceramide } \\
\hline Inflammation & $\begin{array}{l}\text { - } \uparrow \text { Galactosylceramides in GALC mutant mice } \\
\text { - Exogenous galactosylceramide treatment stimulates natural killer T cells }\end{array}$ \\
\hline HIV-1 infection & $\begin{array}{l}\text { - Binding of HIV-1 gp120 to GalCer } \\
\text { - HIV-1 infection in CD4- }{ }^{-} \mathrm{GalCer}^{+} \mathrm{T} \text { lymphocytes }\end{array}$ \\
\hline \multicolumn{2}{|l|}{ Glucosylceramide } \\
\hline Multidrug resistance in cancer cells & $\begin{array}{l}\text { - } \uparrow \text { Glucosylceramides via GCS overexpression in multiple multidrug-resistant tumours and cancer cell } \\
\text { lines }\end{array}$ \\
\hline Inflammation & - $\uparrow$ Glucosylceramides induced by mutations in the $\beta$-glucosylceramidase beta gene (Gaucher disease) \\
\hline Cell adhesion & $\begin{array}{l}\text { - } \uparrow \text { Glucosylceramides via GCS overexpression } \\
\text { - } \uparrow \text { Glucosylceramides in cells treated with } 12 \text { - } O \text {-tetradecanoylphorbol-13-acetate }\end{array}$ \\
\hline Cell differentiation & - $\uparrow$ Glucosylceramides in cells treated with 12 - $O$-tetradecanoylphorbol-13-acetate \\
\hline \multicolumn{2}{|l|}{ Lactosylceramide } \\
\hline Cell proliferation & - $\uparrow$ Lactosylceramides due to upregulation of LCS activity \\
\hline Cell adhesion & $\begin{array}{l}\text { - } \uparrow \text { Lactosylceramides due to upregulation of LCS activity } \\
\text { - Exogenous lactosylceramide treatment }\end{array}$ \\
\hline Angiogenesis & $\begin{array}{l}\text { - } \uparrow \text { Lactosylceramides upon vascular endothelial growth factor treatment } \\
\text { - Exogenous lactosylceramide }\end{array}$ \\
\hline Reactive oxygen species generation & - Exogenous lactosylceramide \\
\hline Inflammation & - Exogenous lactosylceramide \\
\hline
\end{tabular}




\begin{tabular}{|ll|}
\hline Cellular process & Sphingolipid species (and enzymes) involved \\
\hline & $\begin{array}{l}\text { i Lactosylceramides via upregulation of LCS activity in cells treated with lipopolysaccharide and } \\
\text { interferon- } \gamma \\
\bullet \uparrow \text { Lactosylceramides induced by cigarette smoke }\end{array}$ \\
\hline
\end{tabular}

For references see full version of the table in Supplementary information S2 (table). The full version also contains structures of the sphingolipid species. 4-HPR, fenretinide; ACDase, acid ceramidase; ACER, alkaline ceramidase; aSMase, acid sphingomyelinase; bSMase, bacterial sphingomyelinase; C1P, ceramide-1-phosphate; CERK, ceramide kinase; CerS, ceramide synthase; DEGS1, sphingolipid $\delta$ (4)-desaturase DES1; EGF, epidermal growth factor; GALC, galactosylceramidase; GalCer, galactosylceramide; GCS, glucosylceramide synthase; HNSCC, head and neck squamous cell carcinoma; IL-1 $\beta$, interleukin- $1 \beta$; LCS, lactosylceramide synthase; NCDase, neutral ceramidase; nSMase, neutral sphingomyelinase; S1P, sphingosine-1-phosphate; SK1, sphingosine kinase 1; SK2, sphingosine kinase 2; SMS, sphingomyelin synthase; TNFa, tumour necrosis factor $\mathrm{a}$. 